\title{
CONF-9606309--1
}

\section{Grain Boundary Studies of High Temperature Superconducting \\ Materials Using Electron Backscatter Kikuchi Diffraction}

\author{
A. Goyal ${ }^{1}$, E. D. Specht, Z. L. Wang ${ }^{2}$ and D. M. Kroeger \\ Metals and Ceramics Division \\ Oak Ridge National Laboratory \\ P. O. Box 2008, Oak Ridge, TN 37831-6116
}

RECEIVED

JAN 28 १९997

Q.S.TI

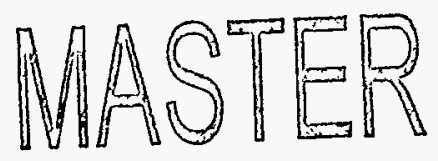

Abstract

The submitted manuscript has been authored by a contractor of the U.S. Government under contract No. DE-AC05-96OR22464. Accordingly, the U.S.

Government retains a nonexclusive, royalty-free license to publish of reproduce the published form of this contribution, or allow others to do so, for U.S. Government purposes."

Grain orientation and grain boundary misorientation distributions in high critical current density, high temperature superconductors were determined using electron backscatter Kikuchi diffraction. It is found that depending on the type of superconductor and the processing method used to fabricate it, there exist different scales of biaxial texture from no biaxial texture, locai biaxial texture, to complete biaxial texture. Experimentally obtained grain boundary misorientation distributions (GBMDs) were found to be skewed significantly to low angles in comparison to what is expected on the basis of macroscopic texture alone, suggesting that minimization of energy may be a driving force during the processing of high critical current density materials. In addition, a higher than expected fraction of coincident-site lattice boundaries is observed. Examination of maps of grain boundary misorientations in spatially correlated grains, i.e. the grain boundary mesotexture, suggests the presence percolative paths of high critical current density. A combination of orientation measurements, theoretical modeling of GBMDs and modeling of percolative current flow through an assemblage of grain boundaries is performed to gain an insight into the important microstructural features dictating the transport properties of high temperature superconductors. It is found that maximization of low energy, in particular, low angle boundaries is essential for higher critical

1 Corresponding author, Ph. No. (423) 574-1587, Fax: (423) 574-7659; Email: ZAG @ ORNL.GOV

2 Now at Georgia Technological University, Atlanta. 


\section{DISCLAIMIER}

Portions of this document may be illegible in electronic image products. Images are produced from the best available original document. 


\section{DISCLAIMER}

This report was prepared as an account of work sponsored by an agency of the United States Government. Neither the United States Government nor any agency thereof, nor any of their employees, make any warranty, express or implied, or assumes any legal liability or responsibility for the accuracy, completeness, or usefulness of any information, apparatus, product, or process disclosed, or represents that its use would not infringe privately owned rights. Reference herein to any specific commercial product, process, or service by trade name, trademark, manufacturer, or otherwise does not necessarily constitute or imply its endorsement, recommendation, or favoring by the United States Government or any agency thereof. The views and opinions of authors expressed herein do not necessarily state or reflect those of the United States Government or any agency thereof. 
currents. The combination of experimental and analytical techniques employed are applicable to other materials where physical properties are dominated by intergranular characteristics. 


\section{Introduction}

Practical applications of high temperature superconductors require fabrication of these brittle, ceramic materials into flexible conductors or wires for most large scale bulk applications like transmission lines, motors, transformers, superconducting magnetic energy storage systems, magnetic resonance systems, etc [1]. A successful method to form conductors has been to fabricate thick films of these materials on flexible silver substrates or by using the oxide-powderin-tube (OPIT) method [2]. In the OPIT process oxide precursor powder is inserted into a silver tube, which is then thermomechanically drawn and rolled into conductor form followed by appropriate heat treatments to form the superconducting phase. While conductors can be made using these techniques with all families of high- $\mathrm{T}_{\mathrm{c}}$ materials, the superconducting interior has a critical current density dependent on the texture and the properties of the grain boundary assemblages formed. So far, the OPIT process has only yielded high critical current densities, $\mathrm{J}_{\mathrm{C}}$, with the $(\mathrm{Bi}, \mathrm{Pb})_{2} \mathrm{Sr}_{2} \mathrm{Ca}_{2} \mathrm{Cu}_{3} \mathrm{O}_{\mathrm{x}}(\mathrm{Bi}-2223)$ compound. The thick film process has primarily been successful with $(\mathrm{Bi}, \mathrm{Pb})_{2} \mathrm{Sr}_{2} \mathrm{Ca}_{2} \mathrm{Cu}_{3} \mathrm{O}_{\mathrm{x}}(\mathrm{Bi}-2212)$ and $\mathrm{TlBa}_{2} \mathrm{Ca}_{2} \mathrm{Cu}_{3} \mathrm{O}_{\mathrm{x}}(\mathrm{Tl}-1223)$ materials. No thermomechanical process has so far yielded high critical currents for the $\mathrm{YBa}_{2} \mathrm{Cu}_{3} \mathrm{O}_{7-\mathrm{x}}(\mathrm{Y}$ 123) compound. Moreover, even in the best conductors of Bi-2223, Bi-2212 and Tl-1223 the transport critical current density is only $1 / 10$ of that achieved in single crystal, epitaxial films of the same materials. Furthermore, magnetically determined intra-granular critical current density is close to that of epitaxial films, suggesting that the $\mathrm{J}_{\mathrm{C}}$ of these conductors is limited by extrinsic defects like grain boundaries.

The various compounds of high- $\mathrm{T}_{c}$ materials not only have widely different transition temperatures $\left(T_{c}\right)$ but also significantly different irreversibility fields $\left(B_{i r r}(T)\right)$ which define the useful range of fields and temperatures for which the materials have high critical current densities in the absence of weak-links. Based on such considerations the Y-123 is the most 
desirable superconducting compound for operations at $77 \mathrm{~K}$ in moderate to high magnetic fields. In order to fabricate conductors of $\mathrm{Y}-123$ and to optimize the properties of $\mathrm{Bi}$-based and $\mathrm{Tl}$ based materials, it is necessary to obtain a better understanding and control of extrinsic defects which dictate the transport properties of these conductors.

The earliest studies of critical current density $J_{c}$ in high- $T_{c}$ oxide superconductors revealed that $\mathrm{J}_{\mathrm{c}}$ for a polycrystalline specimen is much lower than intragranular $\mathrm{J}_{\mathrm{c}}$. These results indicate that for most grain boundaries $\mathrm{J}_{\mathrm{c}}(\mathrm{gb})$, the critical current density for transmission through the boundary, is lower and decreases much more rapidly with field than intragranular $\mathrm{J}_{\mathrm{c}}$. This "weak-link" behavior, which is in contrast to the behavior of low temperature metal superconductors, is attributed to the likelihood that the defect structure and strain associated with the grain boundary are comparable in extent to the superconducting coherence length, which in high temperature superconductors is highly anisotropic and smaller than in metal superconductors. The effects of grain boundary characteristics on current transmission across the boundary have been studied most extensively for $\mathrm{Y}-123$. For clean, stoichiometric grain boundaries, $\mathrm{J}_{\mathrm{c}}(\mathrm{gb})$, appears to be determined primarily by grain boundary misorientation. The dependence of $\mathrm{J}_{\mathrm{c}}(\mathrm{gb})$ on misorientation angle has been determined in (Y123) for grain boundary types which can be formed in epitaxial films on bicrystal substrates. These include [001] tilt, [100] tilt, and [100] twist boundaries [3]. Grain boundaries with misorientation angles larger than about $10^{\circ}$ were found to be weak-links. Recently, the Dimos experiment has been extended to artificially fabricated [001] tilt bicrystals in $\mathrm{Tl}_{2} \mathrm{Ba}_{2} \mathrm{CaCu}_{2} \mathrm{O}_{8}$ [4], $\mathrm{Tl}_{2} \mathrm{Ba}_{2} \mathrm{Ca}_{2} \mathrm{Cu}_{3} \mathrm{O}_{\mathrm{x}}$ [5], Tl1223 [6] and $\mathrm{Nd}_{1.85} \mathrm{Ce}_{0.15} \mathrm{CuO}_{4}$ [5]. In each case it was found that, as in $\mathrm{Y}_{123}, \mathrm{~J}_{\mathrm{c}}$ depends strongly on grain boundary misorientation angle. Although no measurements have been made on the Bi-2223, data on current transmission across artificially fabricated grain boundaries in Bi- 
variation in $\mathrm{J}_{\mathrm{C}}$ with grain boundary misorientation in $\mathrm{Bi}-2212$ and $\mathrm{Bi}-2223$ is similar to that observed in the well characterized cases of Y123 and Tl-based superconductors.

In order to determine what makes conductors of Bi-2223 and 2212 and Tl-1223 "tick", studies of grain boundary networks in these materials and how they effect and control percolation of supercurrent were studied using electron backscatter Kikuchi diffraction (BKD). The various types of superconductors were found to have distinct macroscopic and local textures and microstructures. Grain boundary misorientation distributions (GBMD) were highly dependent on the compound and the method used for fabrication. Comparison of experimentally obtained GMBDs to theoretically expected GBMDs based on texture alone, indicated that in each case the population of low energy boundaries was much higher than expected. Modeling of current flow for the various types of microstructures was performed to determine optimum microstructures and guide processing. Results of this study have led to the development of a new method to form macroscopically, biaxially textured superconductors. The particular methods used to characterize and model microstructures are equally applicable to other materials and therefore have broad applications.

\section{Experimental}

\subsection{Bi-2223 Conductors}

High critical current density conductors were obtained from American Superconductor Corporation. Multifilamentary Bi-2223 conductors for microstructural analysis were prepared using standard oxide powder-in-tube techniques [9]. Oxide powders with an initial metals ratio of 1.7:0.4:1.9:2.1:3.1 (Bi:Pb:Sr: $\mathrm{Ca}: \mathrm{Cu}$ ) were used. These powders were packed into Ag billets, which were subsequently deformed to hexagonal rods using extrusion, swaging and drawing 
techniques. Nineteen of these hexagonal rods were bundled together and inserted into a $\mathrm{Ag}$ tube. The resulting composite bundle was drawn and then rolled to a tape. An iterative thermomechanical process was used to optimize the superconducting properties of the tape. Heat treatments were performed between $800-830^{\circ} \mathrm{C}$ in $0.0075 \mathrm{~atm} \mathrm{O}$, the thermodynamic stability field of the Bi-2223 phase. Rolling was used to densify the tape in between heat treatments. Fully processed tapes are near $100 \%$ dense and have a transport critical current density in the range of $10-20 \times 10^{3} \mathrm{~A} / \mathrm{cm}^{2}$ at $77 \mathrm{~K}$ in self-field using a $1 \mu \mathrm{V} / \mathrm{cm}$ criterion. Individual filaments in the tape are about $20 \mu \mathrm{m}$ thick and $200 \mu \mathrm{m}$ wide. To facilitate microstructural analysis, $\mathrm{Ag}$ was chemically etched from one side to reveal the superconductor. The etching procedure does not interact with the superconductor. Figure 1 shows a scanning electron image (SEM) image of a filament surface. Most grain boundaries are clearly visible, permitting the determination of electron back scatter diffraction patterns from adjacent grains. Diffraction patterns were obtained for all of the grains which could be distinguished in several small areas containing from 15 to $\sim 100$ contiguous grains [10]. Since the surface of the sample is not flat, good patterns are not obtained at most locations. Manual location of the bands was found to be necessary to obtain accurate indexing of the patterns.

\subsection{Bi-2212 Thick Films}

The highest critical current density films of this compound made by Kumakura et al. at the National Research Institute for Metals, Japan were examined [11]. Experimental details for film are fabrication have been reported by them [11]. 


\subsection{Tl-1223 Thick Films}

The highest critical current density films made by Deluca et al. [12] at General Electric Corporation were examined. Experimental details for film fabrication have been reported by them. Briefly, precursor materials comprising of $\mathrm{Ba}_{2} \mathrm{Ca}_{2} \mathrm{Cu}_{3} \mathrm{O}_{x}$ are spray deposited onto a polycrystalline yttria-stabilized-zirconia substrate (YSZ) followed by vapor phase incorporation of $\mathrm{Tl}$ in a two zone Tl-reactor.

\subsection{BKD Data Analysis}

Data was obtained on various scanning electron microscopes (SEMs), a Cambridge 800 SEM fitted with a fibre optically coupled silicon intensified target camera (SIT) and a Philips XL30 Field-Emmission SEM fitted with a fibre optically coupled SIT. Since all the materials examined were strongly c-axis textured, the dominant pole in the diffraction pattern exhibited 4fold symmetry and was indexed as the [001] pole of the approximately tetragonal subcells. Figure 2 shows a typical backscatter diffraction pattern from Bi-2223. From the indexed diffraction pattern, the absolute orientation of a grain was determined. Grain boundary misorientation between two contiguous grains A1 and A2, were calculated using Bollman's theory, $\mathrm{M} 12=\mathrm{A} 1^{-1} \mathrm{~A} 2$ [13]. Depending on the crystal symmetry, the angle/axis of misorientations has many symmetrically equivalent forms ( 24 for cubic, 8 for tetragonal etc); and the variant containing the smallest positive misorientation angle was chosen as the description of the disorientation.

3. Results and discussion 
3.1. Bi-based materials

3.1.1. Bi- 2223

Figure $3 \mathrm{a} \& \mathrm{~b}$ summarize orientation measurements from 113 spatially correlated grains of Bi2223. As indicated in the (001) pole figure shown in Figure 3a, excellent c-axis texture exists. However no in-plane texture is observed as illustrated by the pole figure shown in Figure $3 \mathrm{~b}$. Fig. 4a shows the proportion of small angle and coincident site lattice boundaries found in this area containing 113 grains and resulting in 227 identifiable boundaries. More than $40 \%$ of the boundaries are $\Sigma 1$ boundaries, i.e., have misorientation angles less than $15^{\circ}$ and $30 \%$ of the boundaries have misorientations less than $10^{\circ}$. Small angle boundaries have low energies and can be expected to be less detrimental to $\mathrm{J}_{\mathrm{c}}$ than high angle boundaries. In addition, $8 \%$ of the boundaries were found to be within the Brandon criterion for CSLs larger than 1 and less than 50 and with rotation axis [001]. The boundaries were classified as low sigma if the composite axis/angle deviation from the exact sigma orientation was within the Brandon criterion [14]. The deviations were calculated using a method discussed by Bollman [13] and Zhu et al.[15]. However, the properties and therefore the importance of coincidence site lattice (CSL) boundaries have not been determined, although there are indications that some CSL boundaries may be better than other large angle boundaries $[7,8]$. The data in Fig. 4a suggests that their numbers may be only slightly enhanced. Fig. $4 \mathrm{~b}$ shows the frequency of CSL boundaries with the $\Sigma$ less than 50 .

The large fraction of low angle grain boundaries suggests that percolative paths for strongly linked flow may exist. Examination of maps of misorientation angles over small areas indicates that two-dimensional percolative paths among grains at the $\mathrm{Ag} /$ superconductor interface can 
indeed be found. Fig. 5 shows the spatial distribution of grain boundaries in a region of the BSCCO tape. Small angle boundaries are marked by dashed lines, solid lines designate large angle boundaries and dot-dash lines indicate those boundaries that are within the Brandon criterion for a CSL. Many small angle boundaries are present in this region. Percolative paths consisting of low angle boundaries can easily be traced through the microstructure from the bottom left to the top right in Fig. 5 . Since the c-axes are not perfectly aligned, most boundaries have a mixed character, i.e. have both tilt and twist components. Mixed boundaries which have a significant $\mathrm{c}$-axis misalignment component and still have a small, absolute misorientation angle (in other words have small basal plane misorientations), provide opportunities for the current to percolate in the thickness direction of the tape, assuring three-dimensional current flow. An example of such a boundary is at the top left corner of Fig. 5. Such a mechanism for three dimensional current flow is important since c-axis conduction is very limited in the Bi-based materials due to their highly anisotropic crystal structure [16,17].

In addition, it is important to note that the samples contain many "bent" grains. By moving the electron probe within grains which morphologically appear "bent" in the SEM image, it is found that in many such grains the (001) pole in the EBSP pattern changes continuously and not abruptly. This indicates that the bent grains contain a series of boundaries which accommodate the change in orientation without the presence of any large angle boundary. It is surprising to find that such low angle boundaries are not replaced by a high angle boundary after the final extended high temperature anneal that is given to the sample, i.e., the typical polygonization process observed in metals does not occur. Also, recent nanoindentation studies of Bi-2212 and Bi-2223 powder-in-tube samples reveal that these materials possess a unique deformation behavior [18]. This is dominated by extensive cleavage on (001) planes at small applied loads. Once cleaved into thin sheets, individual sheets are highly flexible, and their deformation characteristics are similar to those observed for mica [18]. The typical deformation processing 
schedule that is normally used to fabricate these tapes can be expected to result in numerous bent grains. Such bent grains can easily be found in metallographic examination of transverse crosssections of these tapes. If many such bent grains accommodate the change in orientation comprising the bend by a series of small angle boundaries as observed here, then they provide an important mechanism for current flow between c-axis misaligned grains and further ensure three dimensional current flow in the tapes.

\subsubsection{Bi-2212}

Thick films of $\mathrm{Bi}-2212$ show a macroscopic texture similar to that of $\mathrm{Bi}-2223$, however there are differences in the GBMDs. Data was collected from many spatially correlated grains resulting in 300 grain boundaries. The total percentage of small angle boundaries was found to be $33 \%$. The percentage of CSL boundaries larger than 1 and less than 50 was found to be $25 \%$, significantly higher than that observed in Bi-2223. Figure 6 shows the population of CSLs observed in the sample. A higher population of CSLs in this material is consistent with the significantly larger amounts of liquid phases present during the processing of this material compared to that of $\mathrm{Bi}$ 2212.

Some samples of Bi-2212 were alșo found to be flat enough to perform orientation imaging (OIM) scans. Figure 7a \& b show OIM scans from a region of the film $\sim 400 \mu \mathrm{m} \times 140 \mu \mathrm{m}$. Data was taken on a hexagonal grid of $2 \mu \mathrm{m}$ spacing. Two sets of grain boundaries are drawn, the thinner boundaries indicate misorientations greater than $5^{\circ}$ and less than $15^{\circ}$. The thick boundaries indicate misorientations larger than $15^{\circ}$. In Figure $7 \mathrm{a}$, a given color represents percolative regions within $2^{\circ}$. Figure $7 \mathrm{~b}$ shows the same region with a given color now representing percolative regions within $15^{\circ}$. Clearly some evidence of "local" biaxial texture is observed. 
3.1.3. Expected GBMDs as a function of c-axis texture in $\mathrm{Bi}-2223$ and $\mathrm{Bi}-2212$

Computer simulation of a model polycrystal was performed by generating a set of 200 grains, the crystallographic orientations of which were described using Euler angles $\phi_{1}, \phi$ and $\phi_{2}$ [19]. These Euler angles define a rotation matrix $g$ which defines the orientation of a crystallite in the model polycrystal with respect to the specimen or global coordinate system. The notation similar to that defined by Bunge [20] was used. Each grain has a random orientation in relation to a global polycrystal basis $\mathrm{G}$ [e1,e2,e3]. There is linear dependence between the crystal coordinate basis, $\mathbf{C}[\mathrm{f} 1, \mathrm{f} 2, \mathrm{f} 3]$ and basis $\mathbf{G} ; \mathbf{C}=\mathrm{g} . \mathbf{G}$, where $\mathbf{g}$ is an appropriate orthogonal transformation matrix determining the orientation of the crystal in the global basis $\mathbf{G}$. The matrix $\mathbf{g}$, determined from the Euler angles describes three successive rotations $\mathbf{R}$, which describe the final orientation of the crystal coordinate system, $\mathrm{C}$, with respect to the sample coordinate system $\mathrm{G}$. If two grains 1 and 2 have orientations $\mathrm{g} 1$ and $\mathrm{g} 2$ respectively, then their mutual misorientation angle is described by the rotation matrix $\mathrm{g} 12=\mathrm{g} 1^{-1} \mathrm{~g} 2$. The geometrical characteristics of the boundary, i.e., the angle and axis of misorientation (the so called disorientation), is extracted from the properties of the misorientation matrix.

Computer simulation of a random distribution of grain boundaries was performed by randomly assigning three Euler angles in the ranges specified above to each grain using a standard random number generator, and then determining misorientations of all possible grain boundaries that can be formed from the set. Since 200 grains were used, this results in $\sim 20000$ grain boundaries. Figure $8 \mathrm{a}$ shows the distribution of misorientation angles obtained from the simulation of a randomly oriented cubic polycrystal using this technique. The shape of this histogram is in good agreement with theoretical estimates of Mackenzie [21]. The fraction of grain boundaries with 
misorientations less than a given angle can be obtained by integrating and normalizing Figure $8 \mathrm{a}$, and is shown in Figure 8b (solid curve). Similar curves are also shown for tetragonal and orthorhombic materials. With decrease in crystal symmetry, the fraction of low angle boundaries decreases as expected. Since the simulated polycrystal is random, in each case the population of small angle boundaries is small.

The effect of texture was studied by restricting the values of Euler angles to certain specific ranges. For example, to study the effect of c-axis texture, only the value of $\phi$ was restricted to various values. In order to study the effect of biaxial texture, values of only two Euler angles $\phi_{1}$, $\phi$ need to be restricted when is $\phi$ small. Figure 9 shows the effect of macroscopic c-axis texture on the misorientation angle distribution for tetragonal materials. The fraction of misorientation angles less than an angle $\phi$ is plotted for cases in which the c-axes of the grains were restricted to ranges of $10^{\circ}, 20^{\circ}, 30^{\circ}$, etc., and the a-axis are random and not restricted. The straight line denotes what would be expected for perfect c-axis texture. It is evident from the figure that the fraction of small angle boundaries increases with better c-axis texture. The number of small angle boundaries increases as the range of variation of the c-axis orientation is decreased. For perfect $\mathrm{c}$-axis alignment, $22.2 \%$ of boundaries would have misorientation angles less than $10^{\circ}$. Bi-2223 powder-in-tube conductors typically have $\mathrm{c}$-axis rocking curves with full-width-at-halfmaximum $\sim 15-20^{\circ}$, suggesting that the $10^{\circ}$ or $20^{\circ}$ curves in Fig. 9 may apply to them.

\subsubsection{Effect of c-axis Texture and Dimensionality on Critical Current Density}

For a tetragonal material with perfect c-axis alignment, there are no c-axis tilted grains to carry the current, hence, transport can be expected to be two-dimensional, with a bond percolation threshold of 50\% for an FCC lattice [22]. Even for the most favorable 2D case of a hexagonal lattice, the percolation threshold is $35 \%$, so the material is near or below the percolation 
threshold and high- $\mathrm{J}_{\mathrm{c}}$ is not possible. The situation is at the other extreme for a completely untextured material. Current flow is truly three-dimensional, so percolation will occur when only $12 \%$ of the grain boundaries are conducting (if the grains were arranged in a FCC lattice). However, for a randomly oriented tetragonal material, only $3 \%$ of the grain boundaries have misorientations below a critical misorientation angle $\theta_{c}$ of $10^{\circ}$, and so current cannot flow through low-angle boundaries in this limit either. This suggests that there exists a $c$-axis texture which lies between these two limits for which current flow is optimized. A numerical model addressing this problem was developed by Specht et al. [23]. Figure 10 summarizes the results of the calculations and show the in-plane and out-of-plane $J_{\mathcal{c}}$ normalized to the intragranular $J_{\mathcal{c}}$ as a function of c-axis full-width-half-maximum (FWHM). An optimum c-axis FWHM exists for a given $\theta_{c}$. The effect of a different critical misorientation angle, $\theta_{\mathcal{c}}$, above which $J_{\mathcal{C}}$ is low, is also shown in Figure 10. In general, with increase in $\theta_{c}$, the optimum c-axis alignment increases to higher FWHM. However, it is likely that $\theta_{\mathrm{c}}$ for bi-based materials will be close to $10^{\circ}$ as is the case with other high- $T_{\mathfrak{c}}$ materials.

In an effort to improve the properties of $\mathrm{Bi}-2223$ conductors, processing was aimed at obtaining conductors with better c-axis alignments. Figure 11 shows an SEM image of a region of tape examined. Color-coded grain boundaries are superimposed on the micrograph. Red denotes high angle boundaries, green low angle boundaries, dashed red lines indicate boundaries within the Brandon criterion for a CSL, and dashed lines with a dot indicate those boundaries which are within $1^{\circ}$ of a CSL boundary. Figure 12a shows the statistics for grain boundaries in this region and Figure $12 b$ shows the frequency of CSL boundaries. Again a very high fraction of low angle boundaries is found. The $c$-axis alignment of this tape was $\sim 12^{\circ}$, much sharper than results of the tape presented earlier. This appears to significantly increase the fraction of CSL boundaries significantly. Similar populations of low angle boundaries are found as presented earlier. 
Further increase in the properties of these materials will occur by better control of the processing to obtain materials with optimum microstructures.

\section{2. $\mathrm{Tl}-1223$}

We have reported in the past that thick films of Tl-1223 are composed of "colonies" of locally biaxially aligned grains [24]. All the colonies are uniaxially aligned with respect to their c-axis, which is also perpendicular to the polycrystalline YSZ substrate on which the films are grown. However, little or no in-plane texture is observed between the colonies. Figure 13 shows a typical microstructure of the films. Figure 14 shows the typical grain orientation structure and texture within a colony as determined by electron backscatter Kikuchi diffraction [25]. Figure 14 was obtained by moving the electron beam continuously across the sample in straight lines while recording the instantaneous backscatter diffraction pattern. Post processing of the data was then performed to locate the position and nature of the grain boundaries. Since the c-axis of all the grains in the film is aligned perpendicular to the film, grain orientations in the tetragonal Tl1223 film can be represented by a single arrow indicating the location of the a-axis. Arrows are placed at the location of the grain boundary by projecting the a-axis of the grain on to the substrate surface (i.e. the R-T plane). The arrow represents the orientation of the grain to the right of the boundary. During the measurement the substrate sides were aligned along parallel and perpendicular to the specimen co-ordinate system and correspond to the $\mathrm{R}$ and $\mathrm{T}$ directions. Due to the four-fold symmetry for the tetragonal structure about the c-axis, the angle in the R-T plane was chosen to lie within the first quadrant, i.e. between $0-90^{\circ}$. As shown in Figure $14 \mathrm{~b}$, colonies are comprised of well-aligned grains. A (100) pole figure corresponding to the "arrow" map is shown in Figure 14a. The presence of a single colony of aligned grains is evident. Calculation of grain boundary misorientations between neighboring grains in this $40 \times 120(\mu \mathrm{m})^{2}$ 
region shows that $91 \%$ of the boundaries have misorientations less than $15^{\circ}$ and $80 \%$ less than $10^{\circ}$. X-ray phi scans obtained from a single colony give rise to a single peak for a $90^{\circ}$ rotation of the sample about the c-axis. The FWHM of the peak then represents the "spread" in orientations within a colony. The distance between two adjacent arrows is representative of the grain size and on average is in the range of $5-10 \mu \mathrm{m}$.

The structure of typical colony intersections are indicated in Figures 15 and 16. Figure 15a shows the (100) pole figure of a region comprising two colonies where the average misorientation between the colonies is large. Figure $15 \mathrm{~b}$ shows the grain orientation structure at the colony intersection. The population of boundaries in this region with misorientation angles less than $15^{\circ}$ is $83 \%$ and less than $10^{\circ}$ is $75 \%$. This is not surprising because most grain boundaries within a colony are small angle and high angle boundaries are formed primarily at the colony intersection. What is further interesting is that $54 \%$ of the high angle boundaries at the colony intersection are within the Brandon criterion [12] for CSL boundaries with $\Sigma<50$. Figure 16 a shows the (100) pole figure of another region comprising two colonies. While the average misorientation between the two colonies in this area is also large $\left(>15^{\circ}\right)$, the misorientation is accommodated by a continuous change in orientation across the intersection as shown in Fig.16b. The population of boundaries in this region with misorientation angles less than $15^{\circ}$ is $63 \%$ and less than $10^{\circ}$ is $51 \%$. In addition $14 \%$ of the high angle boundaries are within the Brandon criterion for CSL boundaries with $\Sigma<50$. Regions as large as $1000 \times 500$ $(\mu \mathrm{m})^{2}$ have been mapped using this technique and over 1500 grain boundaries have been characterized. In general a large fraction of low angle boundaries is found ( $60 \%$ on average). Larger regions are more complex and contain a mixture of the three cases shown in Figure 1416. Figure $17 \mathrm{a} \& \mathrm{~b}$ show the grain orientations in two $1 \mathrm{~mm}$ regions of a film. Figure $18 \mathrm{a} \& \mathrm{~b}$ shows the corresponding (100) pole figures. In Figure 17 the projections of the a-axis of the grains onto the RT plane, i.e. the arrows are color coded such that a given color represents 
orientations within $15^{\circ}$. A colony structure is evident. These regions comprise a total of 1077 grain boundaries. $48 \%$ of these were less than $15^{\circ}, 29 \%$ less than $10^{\circ}$, and $10 \%$ were within the Brandon criterion for a CSL. These particular regions with good alignment were found to have $\mathrm{J}_{\mathrm{c}}$ 's of $1 \times 10^{5} \mathrm{~A} / \mathrm{cm}^{2}$ and $2 \times 10^{5} \mathrm{~A} / \mathrm{cm}^{2}$ respectively.

Since there are only small angle boundaries within a colony, macroscopic current flow is not expected to be well-linked. However, strongly-linked current flow across an aggregate of colony intersections which is contained in a typical film, can be expected to be percolative in nature. Assuming the results from Nabatame et al. [6] are applicable, the current will be expected to weave its way around high angle boundaries, primarily traversing paths comprising connected networks of small angle grain boundaries. One of the consequences of percolative current flow is that the critical current density will depend on the dimensions of the conductor. As the width of the conductor or film is reduced to a dimension comparable to the average colony size in the film, $\mathrm{J}_{\mathrm{c}}$ can be expected to be limited by high angle boundaries due to reduction of percolative options.

Figure 19 shows the most likely a-axis orientations in a $4 \times 5 \mathrm{~mm}^{2}$ region of a $3 \mu \mathrm{m}$ thick Tl-1223 film on YSZ made by spray pyrolysis followed by two-zone thallination. The data was obtained at the National Synchrotron Light Source (NSLS) at the ORNL beam line X-14 [26]. The c-axis is aligned to within $2^{\circ}$ of the normal to the film. X-ray phi-scans were then measured using the $\left\{\begin{array}{lll}1 & 1 & 18\end{array}\right\}$ reflection. Figure 19a shows X-ray $\phi$-scans measured using effective beam sizes of $2 \times$ $11 \mathrm{~mm}^{2}$ (top) and $0.01 \mathrm{~mm}^{2}$ (bottom) respectively. While little in-plane texture is observed with a larger beam, a distinct in-plane texture is observed with the smaller beam. Four peaks are observed for a rotation of $360^{\circ}$ about the sample normal, consistent with the tetragonal symmetry of the material. Figure $19 \mathrm{~b}$ shows a map of a-axis grain orientations measured by 
strongest peak in the first quadrant $\left(0-90^{\circ}\right)$. The crosses indicates the a-axis of the most common orientation in the $\phi$-scan. The colony structure in the sample is evident. It would be intuitively expected that the critical current density of a sample with such a microstructure will be dependent on the bridge dimensions due to change in percolative options by the geometry of the bridge. Such a measurement has been performed and results are in excellent agreement with microstructural predictions $[27,28]$.

\subsubsection{Microstructural Modeling of Current Flow in Tl-1223 Thick Films}

The colony microstructure shown in Figure $19 \mathrm{~b}$ can be described quantitatively by an orientational correlation function, $\mathrm{C}(\mathrm{r}, \phi)=\left\langle\left[\mathrm{I}\left(\mathrm{r}_{\mathrm{o}}, \phi_{\mathrm{o}}\right)-I\left[\left(\mathrm{r}_{\mathrm{o}}+\mathrm{r}, \phi_{\mathrm{o}}+\phi\right)-I\right]\right\rangle_{\mathrm{r}, \mathrm{o}} / I^{2}\right.$, where $I\left(r_{0}, \phi_{0}\right)$ is the diffracted intensity from position $r_{0}$ and orientation $\phi_{0}, I$ is the average measured intensity, and the brackets $\left\langle>_{\mathrm{r}, \mathrm{q}}\right.$ indicate an average over all pairs of measurements separated by a displacement $r$ and misorientation $\phi$ [26]. The critical current density $J_{c}$ can be calculated from the map of orientational distributions in Figure $19 \mathrm{c}$ by the relation $\mathrm{J}_{\mathrm{ij}}=\left\langle\mathrm{I}_{\mathrm{i}}\left(\Phi_{\mathrm{i}}\right) \mathrm{I}_{\mathrm{j}}\left(\Phi_{\mathrm{j}}\right) \mathrm{i}_{\mathrm{c}}\left(\Phi_{\mathrm{i}}-\Phi_{\mathrm{j}}\right)>\right.$ / $I_{\mathrm{i}} I_{\mathrm{j}}$, where the average is over all pairs of angles $\Phi_{\mathrm{i}}$ and $\Phi_{\mathrm{j}}$ [26]. The grain boundary critical current $\mathrm{i}_{\mathrm{c}}$, based on measurements of $\mathrm{Tl}-1223$ films on $\mathrm{SrTiO}_{3}$ bicrystals at $77 \mathrm{~K}$ in zero magnetic [6], is $1 \times 10^{5} \mathrm{~A} / \mathrm{cm}^{2}$ for grains aligned to within 12 and $3 \times 10^{3} \mathrm{~A} / \mathrm{cm}^{2}$ otherwise. The limiting path model $[26,29]$ is used to sum all current paths and find $J_{c}$.

It has been shown [29] that $J_{c}$ for a lattice of randomly oriented grains approaches, for large lattices, the lowest $J_{c}$, i.e. that of a large-angle boundary. A long-range texture increases $J_{c}$ above this minimal value. A colony microstructure is an intermediate case, random overall but locally textured. To see the effect of the colony microstructure on current transport, the $J_{c}$ was simulated for square arrays of colonies, each with random orientation. The grain orientation within each colony follows a Guassian distribution, with the same FWHM for each colony. The 
intercolony $\mathrm{J}_{\mathrm{c}}$ is calculated using the limiting path model, as described above. For large FWHM within a colony, the current transport is limited by the intercolony $\mathrm{J}_{\mathrm{c}}$. This occurs for poor intercolony alignment because while a small fraction of the grains at colony boundaries are well aligned and can carry large currents across the boundary, there is no continuous high-current path across each colony. The intracolony $\mathrm{J}_{\mathrm{c}}$ is simulated by calculating $\mathrm{J}_{\mathrm{c}}$ for a $100 \times 100$ square array of grains. Figure 20 shows the intracolony, intercolony, and total $J_{c}$ for a $100 \times 100$ square array of colonies. As is evident from the figure, $\mathrm{J}_{\mathrm{c}}$ is limited by intercolony transport for low FWHM and by intracolony transport for high FWHM. There exists an optimum FWHM for colonies in order to obtain the highest $\mathrm{J}_{\mathrm{c}}$. This provides processing of these materials a goal to strive towards to obtain higher $\mathrm{J}_{\mathrm{c}}$ ' $\mathrm{s}$.

\subsection{Effect of Biaxial Texture on GBMDs and $J_{c}$}

Comparison of the best Bi-2223 PIT tapes and Tl-1223 thick films studied here, indicate that only a small cross-section of the material, $\sim 10 \%$, is carrying all the current. As shown above, this is due to percolation of current through connected networks of low energy boundaries. Therefore, in order to increase the active cross-section of the material and hence the $\mathrm{J}_{\mathrm{c}}$ of the sample, increased paths of strongly-linked, low energy grain boundaries are required. Fig. 21 shows a calculation of the expected GBMDs when the c-axes of all the grains are perfectly aligned, but the a-axis have varying degrees of alignment. Plotted in the figure is the expected fraction of misorientation angles less than $\Theta$ as a function of $\Theta$ for a tetragonal polycrystal in which the c-axis of all the grains is completely aligned. It is evident from the figure that only a small fraction of biaxial texture greatly increases the population of small angle boundaries. For example, orienting the a-axis of all the grains to within a spread of $50^{\circ}$, results in a grain boundary misorientation with $\sim 50 \%$ of the boundaries less than $15^{\circ}$ and $~ 30 \%$ of the boundaries less than $10^{\circ}$. Even a marginal macroscopic biaxial texture greatly suppresses weak-link 
behavior by enhancing the population of small angle boundaries as was demonstrated for Y-124 PIT fabricated by American Superconductor Corporation [25]. The greatest effect is realized when a sharp biaxial texture is obtained. This is presently possible using two techniques, IonBeam-Assisted-Deposition (IBAD) [30-32] and Rolling-Assisted-Biaxially-Textured-Substrates (RABiTS) [33]. In the IBAD technique, a biaxially oriented buffer layer is deposited using an ion assist gun on randomly oriented metal alloy substrates. Epitaxial YBCO is grown on this layer. In the case of RABiTS, the metal itself is biaxially textured by thermo-mechanical processing, thereby eliminating the IBAD step. Typical in-plane textures obtained by both the techniques are very comparable, $8^{\circ}$ in the RABiT substrate [33] and $12^{\circ}$ in the IBAD buffer layer [31]. In both cases epitaxial YBCO films have yielded high $\mathrm{J}_{\mathcal{C}}$ 's $\left(>10^{5} \mathrm{~A} / \mathrm{cm}^{2}, 77 \mathrm{~K}, 0 \mathrm{~T}\right)$ with field dependence's similar to epitaxial films on single crystal films [30-32].

In the RABiT process a $\mathrm{Ni}$ strip is biaxially textured using thermomechanically processing. Epitaxial metal and oxide buffer layers are then grown on the textured Ni template. The superconductor is grown epitaxially on the last oxide layer. The highest $\mathrm{J}_{\mathrm{c}}$ obtained by this method is $7.1 \times 10^{5} \mathrm{~A} / \mathrm{cm}^{2}$, essentially approaching that of epitaxial films on single crystal ceramic substrates. The key feature of these substrates which allows for such a high $J_{c}$, is the initial grain structure in the Ni substrate. Figure 22 shows three OIM images of these substrates. Patterns were obtained in a hexagonal grid with a step size of $3 \mu \mathrm{m}$. Two types of grain boundaries are indicated in the figures, thin lines denote boundaries with misorientations greater than $5^{\circ}$ and less than $15^{\circ}$, and thick lines are greater than $15^{\circ}$. In Fig 22 , a given color represents a percolative region within $2^{\circ}$ in Fig. 22a, $7^{\circ}$ in Fig.22b and $10^{\circ}$ in Fig.22c. Epitaxial superconducting films on such substrates can also be expected to have a similar grain misorientation structure, comprising primarily of small angle boundaries.

\section{Conclusions}


The results presented here demonstrate for both the Bi-based and Tl-based conductors that small angle grain boundaries are present in much higher numbers than expected statistically from macroscopic textures. These results, in conjunction with the growing body of evidence that large angle boundaries (possibly excluding CSL boundaries) in most, if not all, oxide superconductors are weak-links, strongly suggest that long-range conduction occurs by percolation through a connected network of small angle (and perhaps CSL) boundaries. Critical current density is determined by local grain boundary misorientations, not macroscopic texture. Texture determines the probability that adjacent grains will have similar orientations. Grain boundary energy provides a driving force which skews the misorientation distribution toward small angle and probably CSL boundaries. The specific arrangement of good boundaries comprising the percolative network, i.e., the local texture, varies with material and processing method. In the Tl-1223 deposits a very high density of small angle grain boundaries is associated with the colony microstructure, and long-range conduction depends on percolation through a much smaller population of small angle (and possibly CSL) boundaries at colony intersections. In $\mathrm{Bi}$ 2223 conductor a randomly distributed but smaller population of small angle boundaries is sufficient to ensure percolation of current. It is well-known that $\mathrm{J}_{\mathrm{C}}$ in $\mathrm{Bi}-2223$ conductors does not correlate perfectly with the degree of c-axis alignment and second phase content as determined from x-ray diffraction and microscopic examination of polished cross-sections. We suggest that the population of small-angle boundaries is the "hidden variable" with which $\mathrm{J}_{\mathrm{C}}$ correlates.

Percolation through a connected network of low energy boundaries is offered as a general model for long-range conduction in polycrystalline superconductors. At this stage we have only considered grain boundary misorientation angle as a determinant of $J_{c}(g b)$. It is recognized however that because structure and superconducting properties are anisotropic, the axis of 
rotation and grain boundary plane may also affect $J_{c}(g b)$. Furthermore, there may be an effect of grain boundary structure and energy on chemical stoichiometry of the grain boundary. Large angle boundaries may have greater tendency to form monolayers of second phases or have depletion of chemical contituents like oxygen resulting in a depletion of hole carriers at the boundary.

Microstructural modeling of current flow in uniaxially aligned superconductors suggests that there exists an optimum c-axis alignment for the highest $J_{c}$, due to the combined effects of $J_{c}$ anisotropy and current flow dimensionality. Microstructural modeling of locally biaxially aligned superconductors suggests that there exists an optimum local in-plane alignment for maximum $\mathrm{J}_{\mathrm{c}}$.

In order to increase $\mathrm{J}_{\mathrm{C}}$ of polycrystalline superconductors, optimization of the percolative options is required. Calculation of expected GBMDs for tetragonal materials with varying degrees of biaxial texture suggests that even a small degree of biaxial texture results in a significant increase in the population of small angle boundaries. In an effort to obtain ideal microstructures, macroscopically biaxially textured, flexible metal substrates were fabricated for epitaxial growth of superconductors. Superconductors fabricated on such substrates have $\mathrm{J}_{\mathrm{c}}$ 's approaching those of epitaxial films on single crystal ceramic substrates, and offer a potential route for the fabrication of HTS conductors.

Acknowledgments

We our very grateful to our collaborators: J. Sutliff, J. E. Tkacyzk and J. A. Deluca at General Electric Corporation for useful discussions, collaborations and for providing the T1-1223 samples; G. N. Riley Jr. and M. P. Rupich, American Superconductor Corporation for providing 
the Bi-2223 PIT samples; H. Kumakura, National Research Institute of Metals (NRIM), Japan, for providing the Bi-2212 samples. We are also grateful to D. P. Field and D. J. Dingley, TEXSEM, Utah and T. A. Mason, Idaho National Laboratory, Idaho for fruitful collaborations and discussions. Research sponsored by US Department of Energy, Office of Efficiency and Renewable Energy, Office of Utility Technology - Superconductivity Program and the Office of Energy Research, Basic Energy Sciences, managed by Lockheed-Martin Research Corporation for the US Department of Energy under contract DE-AC05-96OR22464. This research was conducted on part at NSLS, which is sponsored by the US Department of Energy under contract No. DE-AC02-76H00016. 


\section{References}

1. Articles in J. of Metals, Minerals and Materials (Special issue on bulk applications of High- $\mathrm{T}_{c}$ superconductors), vol. 47, No. 8 (1995).

2. Articles in J. of Metals, Minerals and Materials (Special issue on bulk applications of High- $\mathrm{T}_{c}$ superconductors), vol. 46, No. 12 (1994).

3. D. Dimos, P. Chaudhari, J. Mannhart and F. K. LeGoues, Phys. Rev. Lett. 61, 219 (1988); D. Dimos, P. Chaudhari, and J. Mannhart, Phys. Rev. B 41 (1990) 4038.

4. A. H. Cardona, H. Suzuki, T. Yamashita, K. H. Young and L. C. Bourne, Appl. Phys. Lett., 62 (4) (1993) 411.

5. M. Kawasaki, E. Sarnelli, P. Chaudhari, A. Gupta, A. Kussmaul, J. Lacey and W. Lee, Appl. Phys. Lett., 62 (1993) 417.

6. T. Nabatame, S. Koike, O. B. Hyun, I. Hirabayashi, H. Suhara and K. Nakamura, Appl. Phys. Lett. 65 (1994) 776.

7. N. Tomita, Y. Takahashi and Y. Ishida, Jpn. J. Appl. Phys., 29 (1990) L30; N. Tomita, Y. Takahashi, M. Mori and Y. Ishida, Jpn. J. Appl. Phys., 31 (1992) L942.

8. J. L. Wang, X. Y. Lin, R. J. Kelley, S. E. Babcock, D. C. Larbalestier, and M. D. Vaudin, Physica C, 230 (1994) 189. 
9. K. H. Sandage, G. N. Riley Jr. and W. L. Carter, J. Metals, 3 (1991) 21.

10. A. Goyal, E. D. Specht, D. M. Kroeger, T. A. Mason, D. J. Dingley, G. N. Riley Jr., and M. W. Rupich, Appl. Phys. Lett., 66 (1995) 2903.

11. H. Kumakura, H. Kitaguchi, K. Togano, H. Maeda, J. Shimoyama, H. Morimoto, K. Nomura and H. Seido, Advances in Superconductivity IV (1992) 547.

12. J. A. DeLuca, P. L. Karas J. E. Tkaczyk, P. J. Bednarczyk, M. F. Garbauskas, C. L. Briant, and D. B. Sorensen, Physica C 205 (1993) 21; J. E. Tkaczyk, J. A. DeLuca, P. L. Karas, P. J. Bednarczyk, M. F. Garbauskas, R. H. Arendt, K. W. Lay, and J. S. Moodera, Appl. Phys. Lett. 61 (1992) 610.

13. W. Bollman, in "Crystal Lattices, Interfaces and Matrices", published by author, 1982.

14. D. G. Brandon, Acta Metall., 14 (1956) 1479.

15. Y. Zhu, H. Zhang,H. Wang and M. Suenaga, J. Mater. Res., 6 (1991) 2507.

16. B. Hensel, J. C. Grivel, A. Jeremie, A. Perin, and A. Pollini, and R. Flukiger, Physica C. 205 (1993) 329.

17. J. H. Cho, M. P. Maley, J. O. Willis, J. Y. Coulter, L. N. Bulaevskii, P. Haldar, and L. R. Motowidlo, Appl. Phys. Lett. 64 (1994) 3030.

18. A. Goyal. et al., Manuscript in preparation. 
19. A. Goyal, E. D. Specht, T. A. Mason, Appl. Phys. Lett., 68 (1996) 711.

20. H. J. Bunge, in Prefrerred Orientations in Deformed Metals and Rocks: An Introduction to Modern Texture Analysis, edited by H. R. Wenk (Academic, Orlando, FL, 1985), p. 73.

21. J. K. Mackenzie, Acta Metall., 12 (1964) 223.

22. M. F. Skyes and J. W. Essam, Phys. Rev., 133 (1964) A310.

23. E. D. Specht, A. Goyal and D. M. Kroeger, Phys. Rev. B, 53 (1996) 3585.

24. D. M. Kroeger, A. Goyal, E. D. Specht, Z. L. Wang, J. E. Tkacyzk, J. A. Sutliff and J. A. Deluca, Appl. Phys. Lett., 64 (1994) 106.

25. A. Goyal, E. D. Specht, Z. L. Wang, D. M. Kroeger, J. A. Sutliff, J. E. Tkacyzk, J. A. Deluca, L. Masur and G. N. Riley Jr., J. of Electronic Mater., 23 (1994).1191

26. E. D. Specht, A. Goyal and D. M. Kroeger, J. A. Deluca, J. E. Tkacyzk, C. L. Briant and J. A. Sutliff, Physica C, 242 (1995) 164.

27. D. K. Christen, E. D. Specht, , A. Goyal, Q. He, M. Paranthaman, C. E. Klabunde, R. Feenstra, F. A. List, D. M. Kroeger, Z. F. Ren, C. A. Wang and J. H. Wang, Proc. of the 7th US-Japan Workshop on High- $T_{c}$ Superconductors, Oct 24-25, Tsukuba, 1995, pg. 116. 
28. A. Goyal, E. D. Specht, D. K. Christen, D. M. Kroeger, A. E. Pashitski, A.A. Polyanksii and D. C. Larbalestier, To be published in J. of Metals, 1996.

29. J. Rhyner and G. Blatter, Phys. Rev. B 40 (1990) 829.

30. Y. Iijima, N. Tanabe, O. Kohino, and Y. Ikeno, Appl. Phys. Lett. 60 (1992) 769; Y. Iijima, K. Onabe, N. Futaki, N. Sadakata and O. Kohno, J. Appl. Phys. Lett. 74 (1993) 1905.

31. R. P. Reade, P. Burdahl, R. E. Russo and S. M. Garrison, Appl. Phys. Lett. 61 (1993) 2233.

32. X. D. Wu, S. R. Foltyn, P. N. Arendt, W. R. Blumenthal, I. H. Campbell, J. D. Cotton, J. Y. Coulter, W. L. Hults, M. P. Maley, H. F. Safar and J. L. Smith, Appl. Phys. Lett., 1995.

33. A. Goyal, D. P. Norton, J. D. Budai, M. Paranthaman, E. D. Specht, D. M. Kroeger, D. K. Christen, Q. He, B. Saffian, F. A. List, D. F. Lee, P. M. Martin, C. E. Klabunde, E. Hatfield and V. K. Sikka, To be published in Appl. Phys. Lett., 1996; D. P. Norton., Submitted to Science. 


\section{List of Figures}

Fig. 1. a: SEM image of the Bi-2223 surface of a filament revealed by etching away the silver from a multifilamentary powder-in-tube specimen. Grain boundaries are clearly visible, permitting determinations by EBSP of the orientations of contiguous grains.

Fig. 2. Typical backscatter diffraction pattern from a grain of Bi-2223. Since the sample is caxis textured the region near the (001) pole is seen.

Fig. 3. a: (001) pole figure from a region in a Bi-2223 sample. Data was obtained from 113 spatially correlated grains; b: (001) pole figure from the same region.

Fig. 4. a: Statistics for small angle and coincident site lattice boundaries for the region having pole figures shown in Figure 3; b: frequency of CSL boundaries for the same region.

Fig. 5. Spatial distribution of grain boundaries for a region in the Bi-2223 tape. Misorientations are indicated at the grain boundaries, dashed lines denote a small angle boundary $\left(<15^{\circ}\right)$, full lines denote a large angle boundary, and dotted lines designates those boundaries which are within the Brandon criterion for a CSL. Percolative paths consisting primarily of low angle boundaries can be traced from the bottom left to the top right in the SEM micrograph.

Fig. 6. Frequency of CSL boundaries for Bi-2212. Data was obtained from many spatially correlated grains resulting in 300 grain boundaries. 
Fig. 7. OIM images from a region in a Bi-2212 film of size $\sim 400 \mu \mathrm{m} \times 140 \mu \mathrm{m}$. Data was taken on a hexagonal grid of $2 \mu \mathrm{m}$ spacing. Two sets of grain boundaries are drawn, the thinner boundaries indicate misorientations greater than $5^{\circ}$ and less than $15^{\circ}$. The thick boundaries indicate misorientations larger than $15^{\circ}$. In Figure 7a, a given a given color represents percolative regions within $2^{\circ}$. Figure $7 \mathrm{~b}$ shows the same region with a given color now representing percolative regions within $15^{\circ}$.

Fig. 8. a: Distribution of misorientation angles for the 20,000 "grain boundaries" which could be formed among 200 randomly oriented grains in a cubic material. The shape of the histogram agrees well with the calculated curve of Mackenzie [23]; b:Fraction of misorientation angles less than $\Theta$ as a function of $\Theta$, obtained from 20,000 simulated grain boundaries for cubic, tetragonal and orthorhombic symmetries.

Fig. 9. The effect of macroscopic c-axis texture on the misorientation angle distribution from simulations for tetragonal symmetry. In randomly selecting the 200 grain set from which grain boundaries were formed, deviations of the c-axes from perpendicular to the sample plane were restricted as indicated.

Fig. 10. Calculated (a) in-plane and (b) out-of-plane $J_{c}$ normalized to intragranular $J_{c}$ as a function of c-axis texture. An optimum c-axis FWHM exists for a given $\theta_{c}$ where the $\mathrm{J}_{\mathrm{c}}$ begins to drop off.

Fig. 11. SEM image of a Bi-2223 tape with better $\mathrm{c}$-axis alignment. Color-coded grain boundaries are superimposed on the micrograph. Red denotes high angle boundaries, green low angle boundaries, dashed red lines indicate boundaries within the Brandon criterion for a CSL, and dashed red lines with a green dot indicate those boundaries 
which are within $1^{\circ}$ of a CSL boundary. Current paths through low energy boundaries can be traced through most regions of the tape in the area examined.

Fig. 12. a: Statistics for small angle and coincident site lattice boundaries for the micrograph shown in Fig. 11; b: frequency of CSL boundaries in the same area.

Fig. 13.SEM image of the free surface of a polycrystalline $\mathrm{TlBa}_{2} \mathrm{Ca}_{2} \mathrm{Cu}_{3} \mathrm{O}_{8+\mathrm{x}}$ thick film. The rough surface of the sample mandates manual positioning of the beam at points on a grain from where good patterns can be obtained.

Fig. 14. a: (100) pole figure constructed with data obtained from a region $40 \times 120 \mu \mathrm{m}^{2}$ in a T11223 thick film ; b: Arrow map of the projections of a-axes on to the sample plane over the same area. The arrows have similar directions, indicating the area is within a colony. The e-beam was scanned along a horizontal line. When the pattern changed, indicating a grain boundary was traversed, the new grain orientation was calculated and the a-axis projection plotted. The angle/axis pairs were also calculated. For $80 \%$ of the boundaries $\Theta<10^{\circ}$.

Fig. 15. a: (100) pole figure from a region in the Tl-1223 film containing two colonies; b: a-axis arrow map for an area which contains a sharp colony intersection, indicated by the handdrawn curve. For $75 \%$ of boundaries $\Theta<10^{\circ}$, and $54 \%$ of boundaries with $\Theta<15^{\circ}$ are within the Brandon criterion for an ideal CSL.

Fig. 16.a: (100) pole figure from another region in the Tl-1223 film containing two colonies; b: a-axis arrow map for an area in which orientation appears to change gradually without a 
well-defined colony boundary. $52 \%$ of boundaries have $\Theta<10^{\circ}$, and $14 \%$ of large angle boundaries are within the Brandon criterion for an ideal CSL.

Fig. 17.a: Color coded "arrow" maps of a-axis grain orientations in $1 \mathrm{~mm}$ regions of a sample. A given color represents grain orientations within $15^{\circ}$. Data was obtained in the manner described for Figs. 14-16. The colony structure is clearly evident in such macroscopic regions; b: Arrow map of a-axis projections from another region.

Fig. 18. a: (100) and (001) pole figures of orientations indicated in Fig. 17a; b: (100) and (001) pole figures of orientations indicated in Fig. 17b.

Fig. 19. a: Typical $X$-ray $\phi$-scans of c-axis textured Tl-1223 films using effective beam sizes of 2 $x 11 \mathrm{~mm}^{2}$ (top) and $0.1 \mathrm{~mm}^{2}$ (bottom) respectively. While little in-plane texture is observed with the larger beam, a distinct in-plane texture is observed with the smaller beam; b: Map of a-axis grain orientations measured by obtaining $\phi$-scans on a square grid using a $0.1 \mathrm{~mm}^{2}$ beam. The crosses indicate the most common a-axis orientation in the $\phi$-scan.

Fig. 20. Simulated critical current density for $100 \times 100$ arrays of colonies as a function of grain alignment within colonies. $\mathrm{J}_{\mathrm{c}}$ is limited by intercolony transport for low FWHM and by intracolony transport for high FWHM.

Fig. 21. Expected fraction of grain boundary misorientation angles less than $\Theta$ as a function of $\Theta$, for a tetragonal polycrystal with varying degrees of biaxial texture. The fraction of small angle boundaries increases greatly with even a weak biaxial texture. 
Fig.22. OIM images from a macroscopic region of a RABiT substrate. Patterns were obtained in a hexagonal grid with a step size of $3 \mu \mathrm{m}$. Two types of grain boundaries are indicated in the figures, thin lines denote boundaries with misorientations greater than $5^{\circ}$ and less than $15^{\circ}$, and thick lines are greater than $15^{\circ}$. In (a), a given color represents a percolative region within $2^{\circ}$ in (b) a region within $7^{\circ}$ and in (c) a region within $10^{\circ}$. Percolation through small angle boundaries can clearly be envisaged. 
son

w. (W)

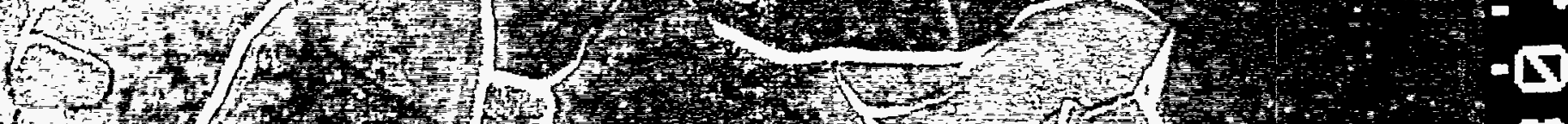

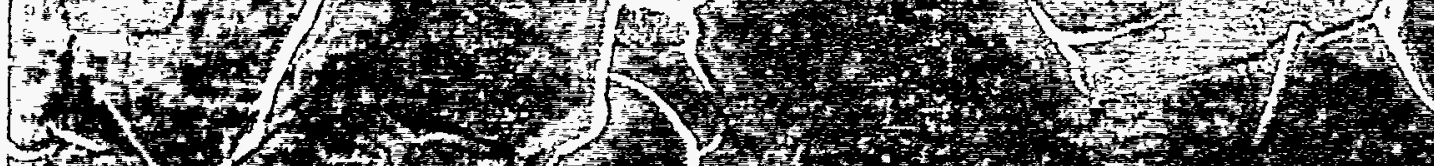

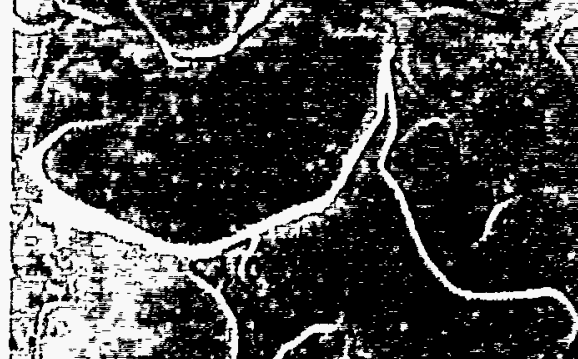

10 2

(
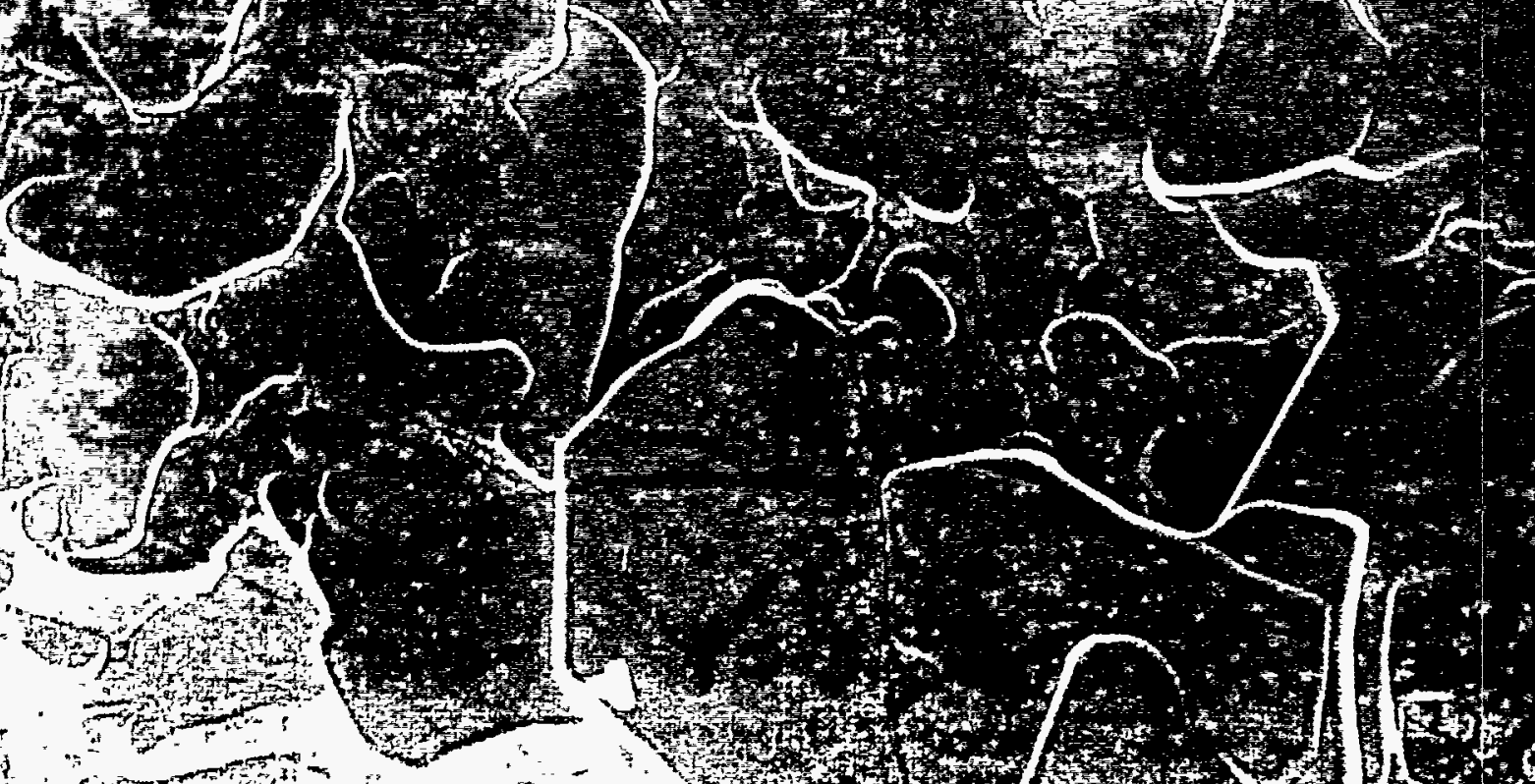

Y

1)

1.

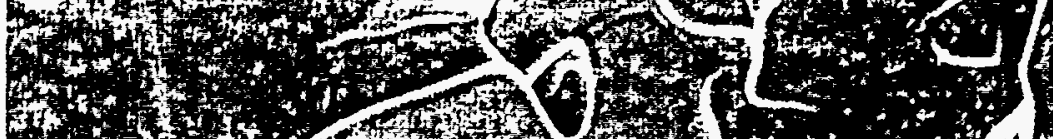

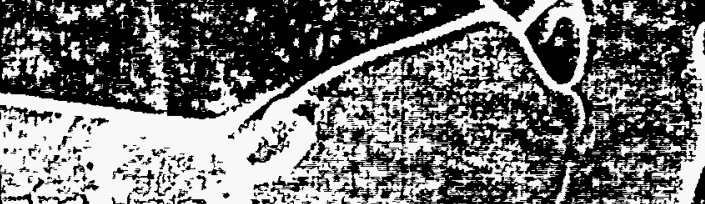

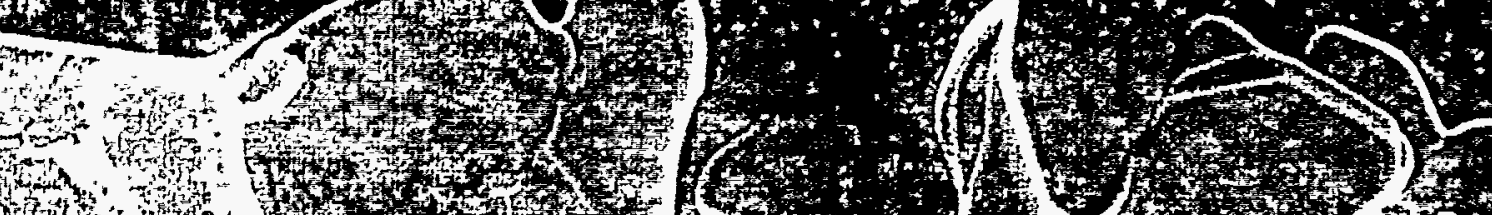

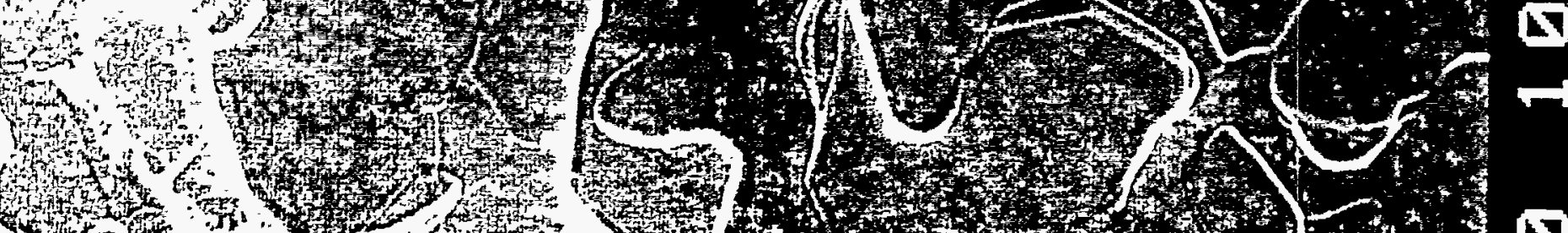
\& Q6) mo

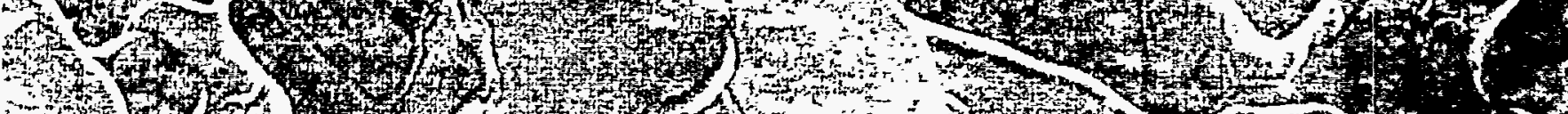

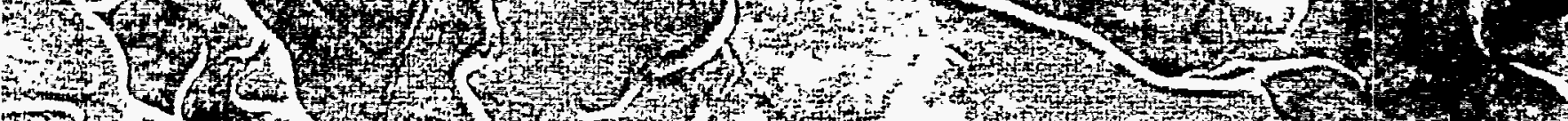

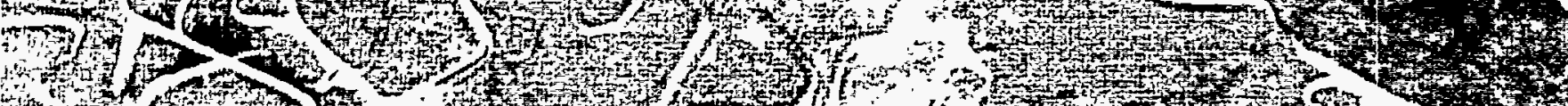

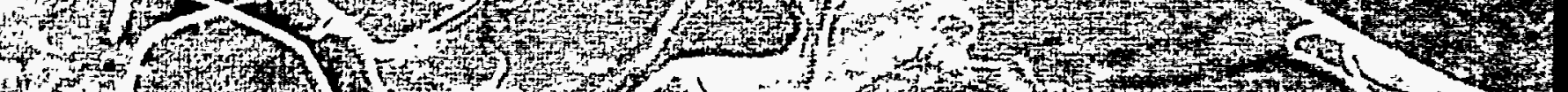
(17) 


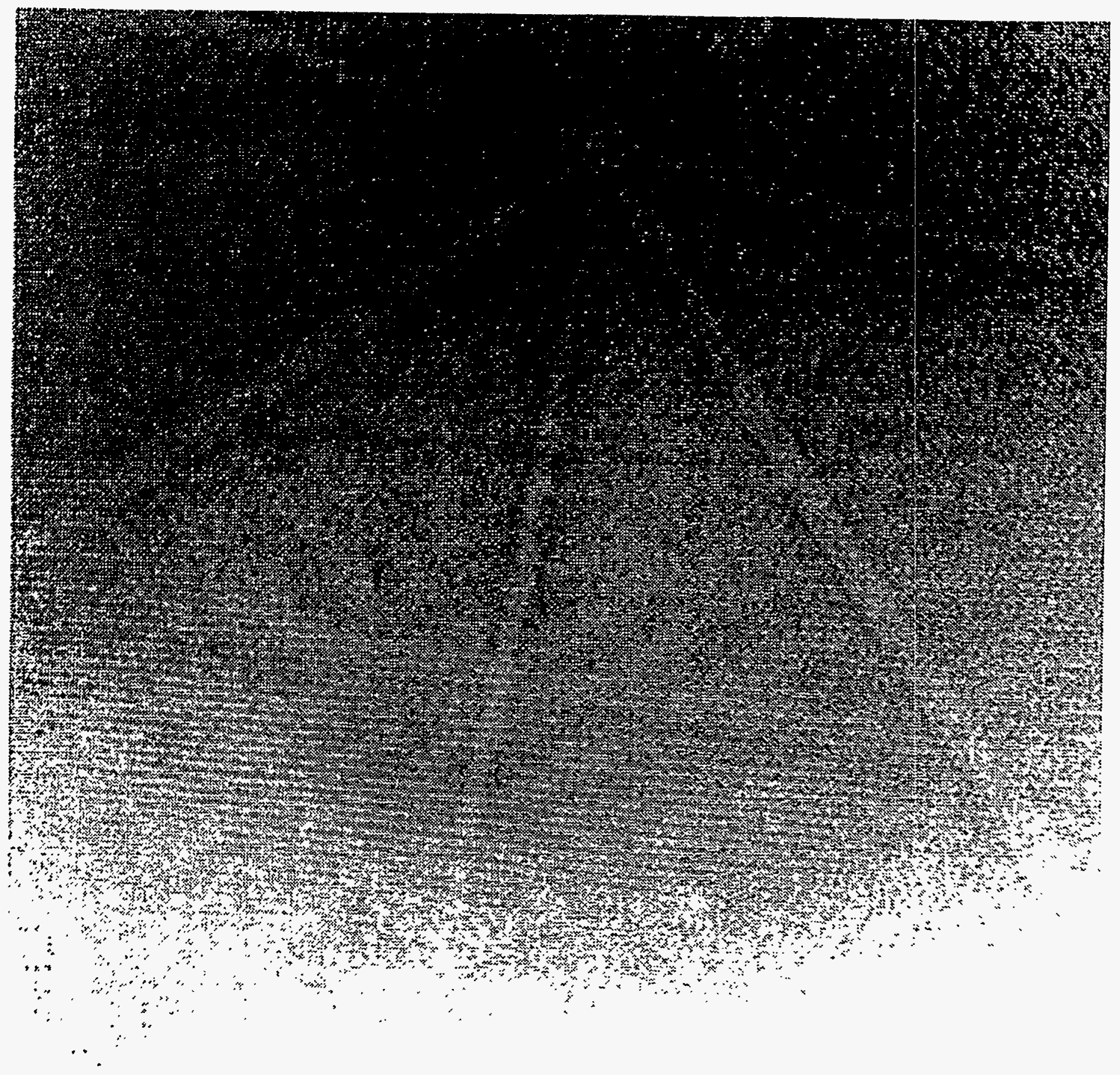



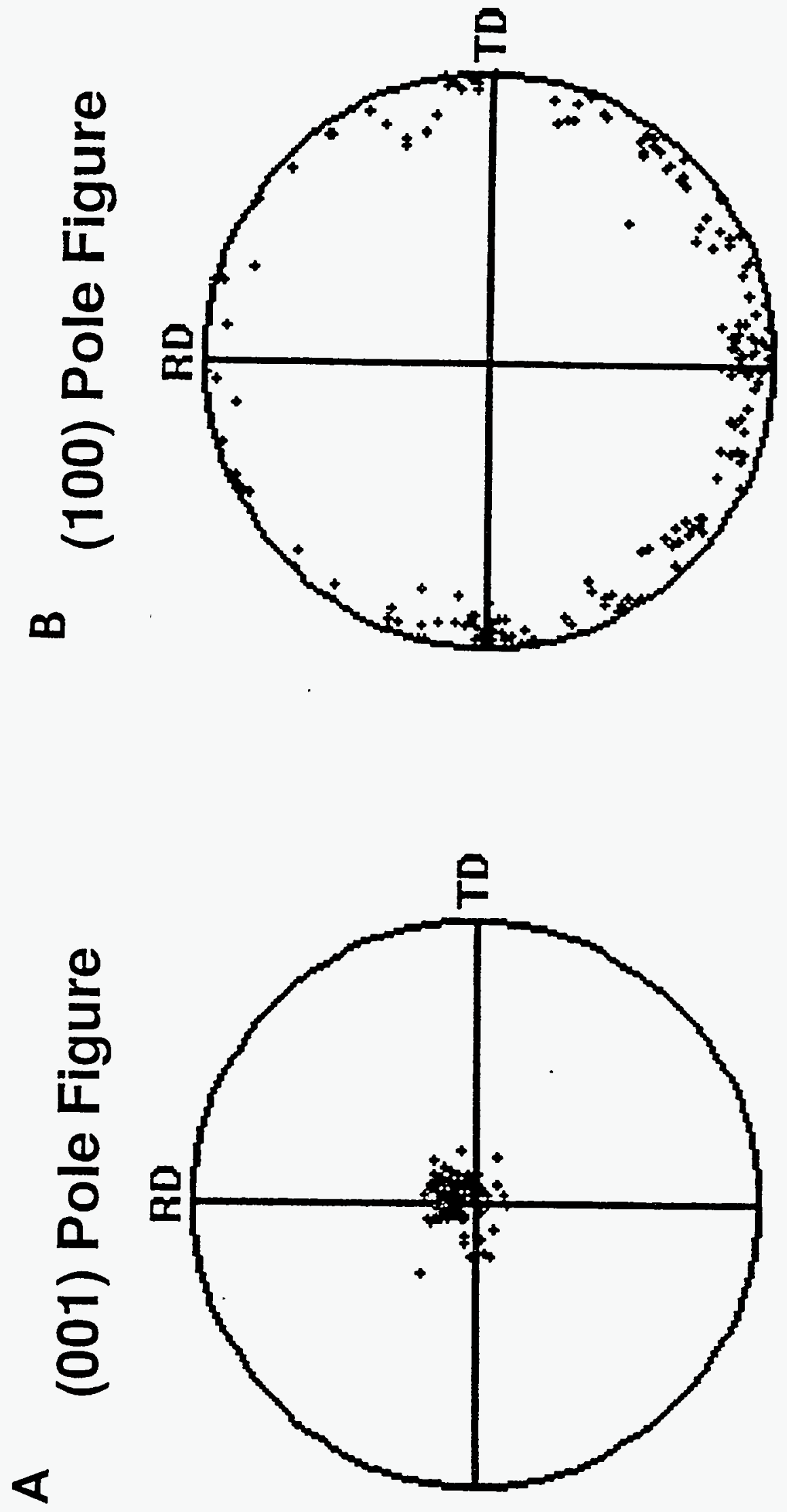

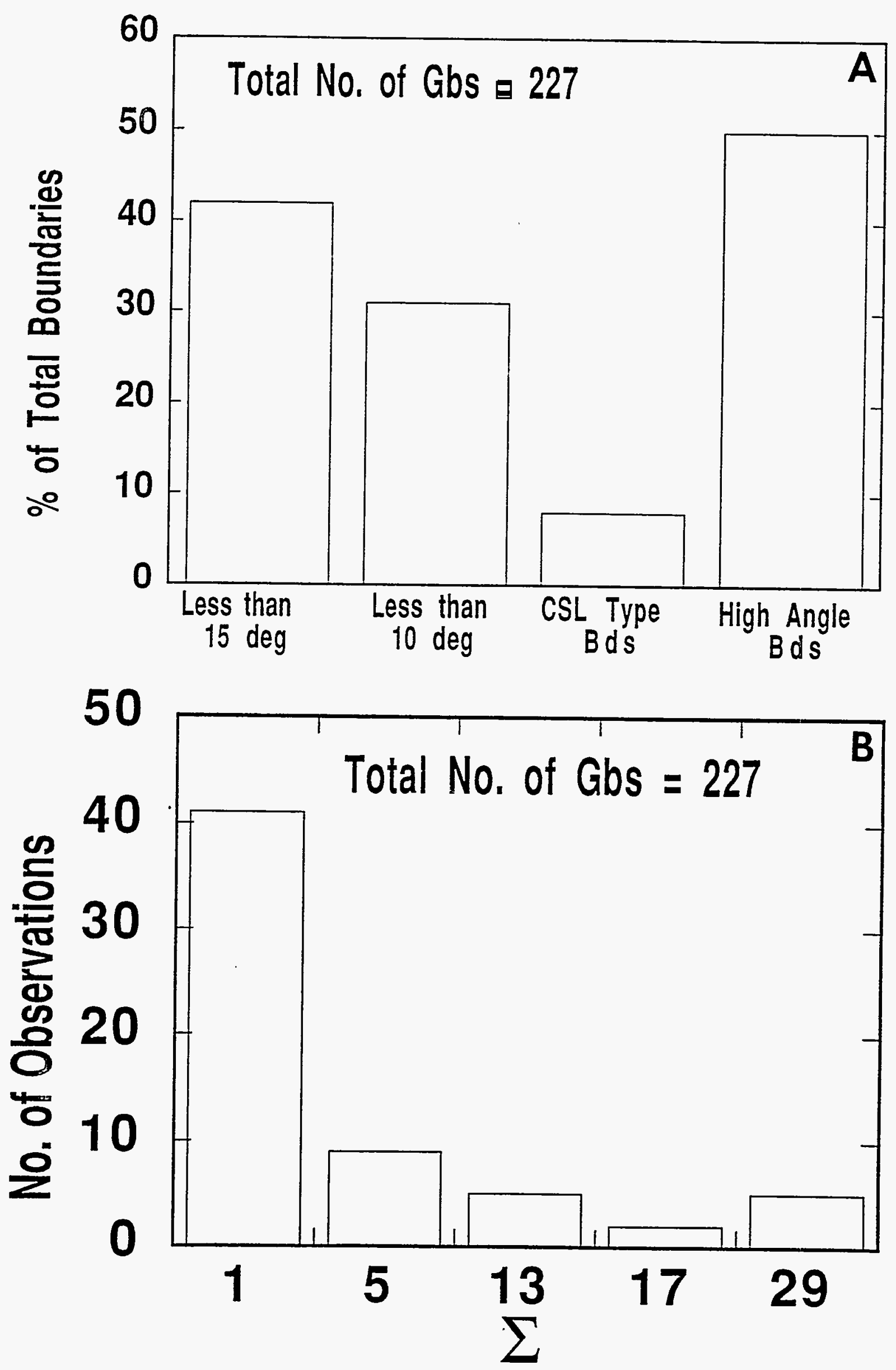


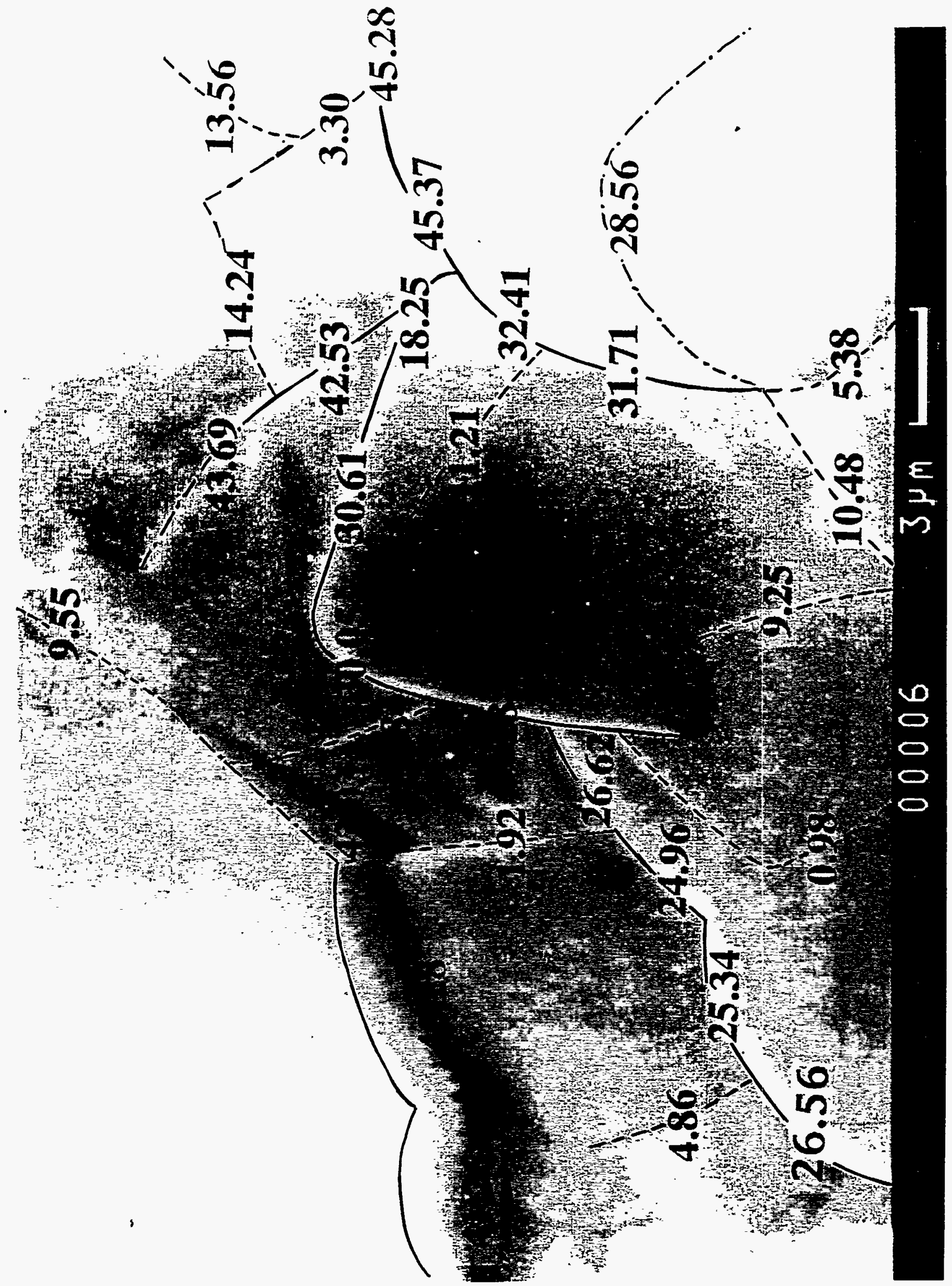




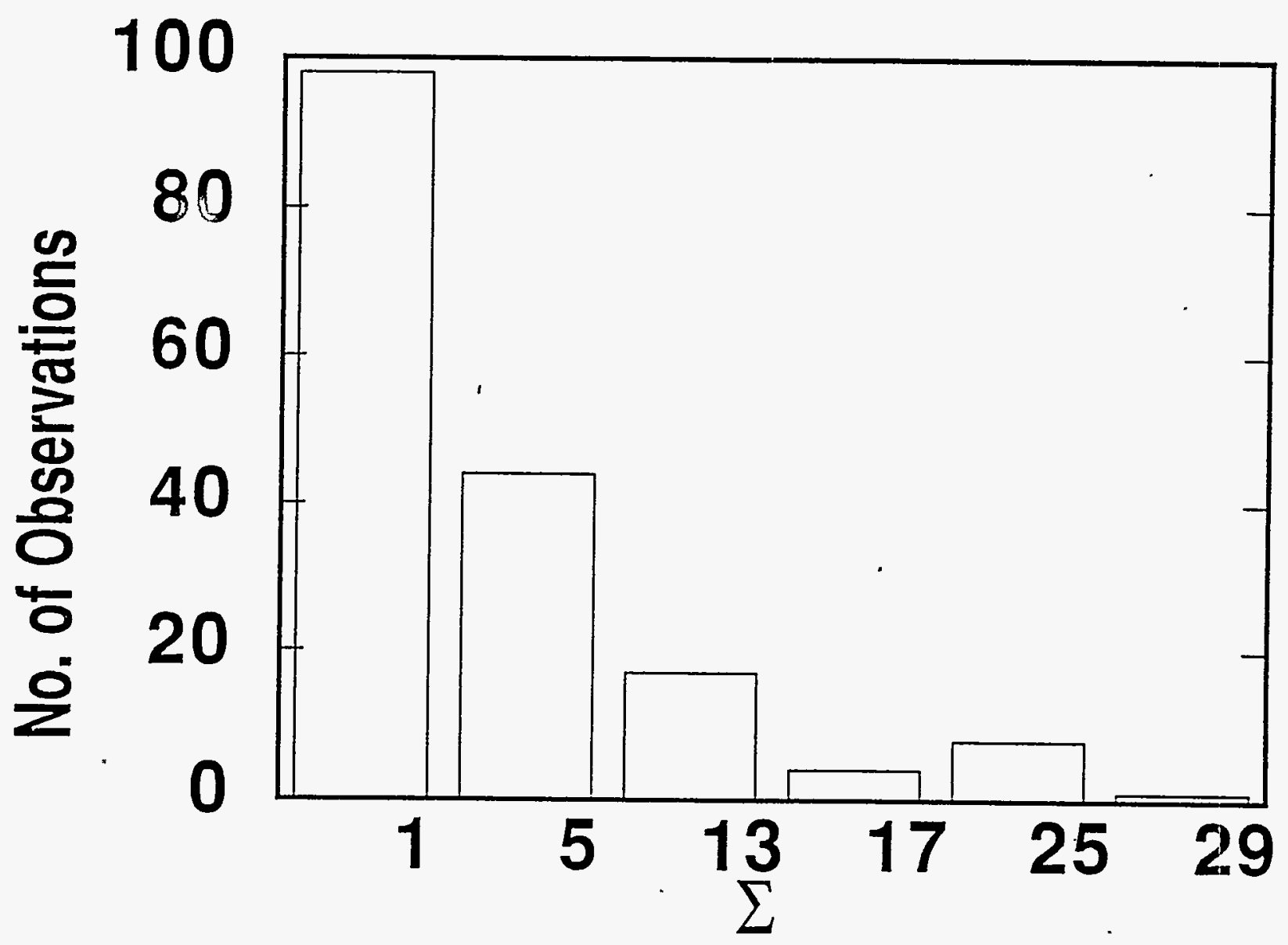



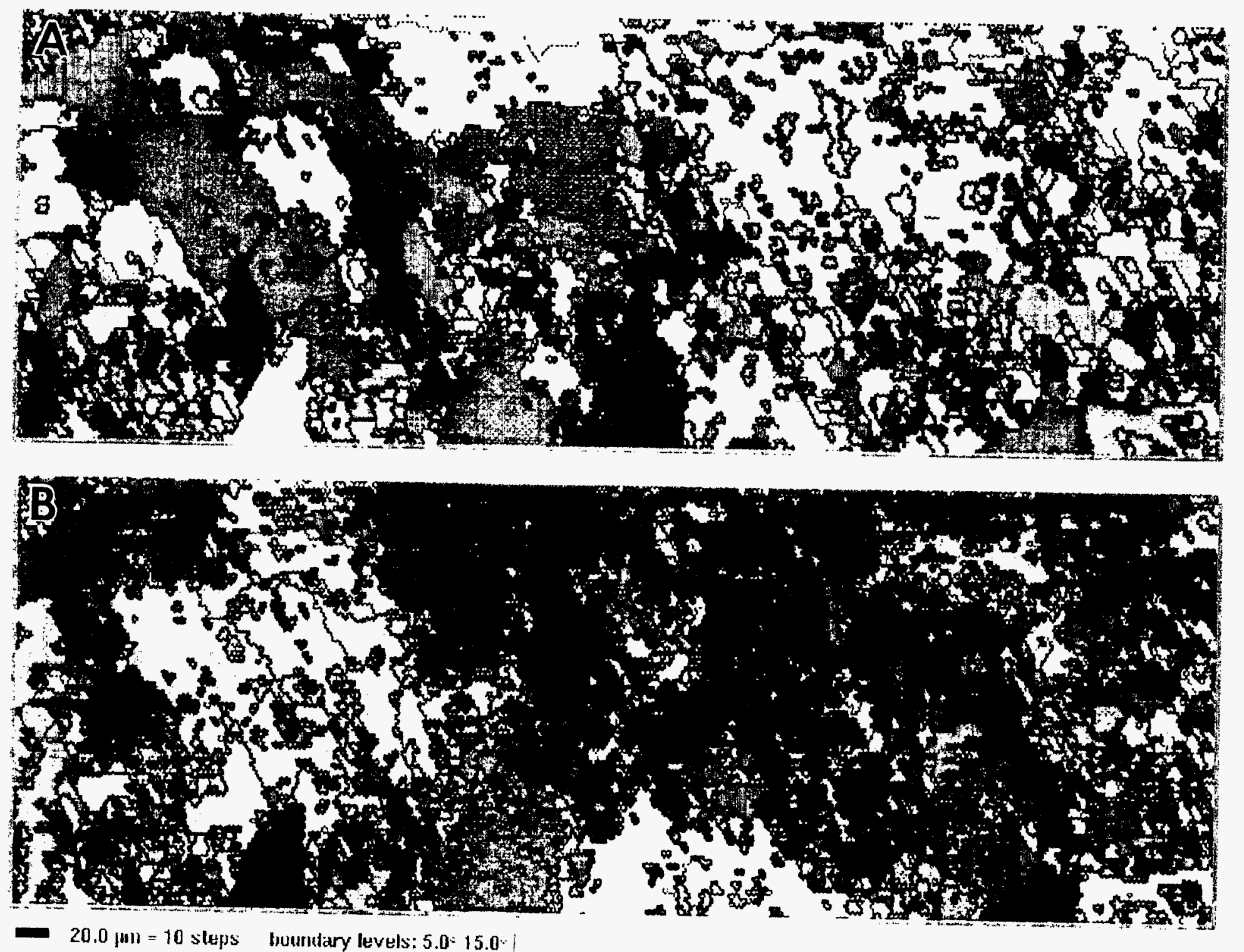
Fraction of Misorientation Angles Less than $\Theta$

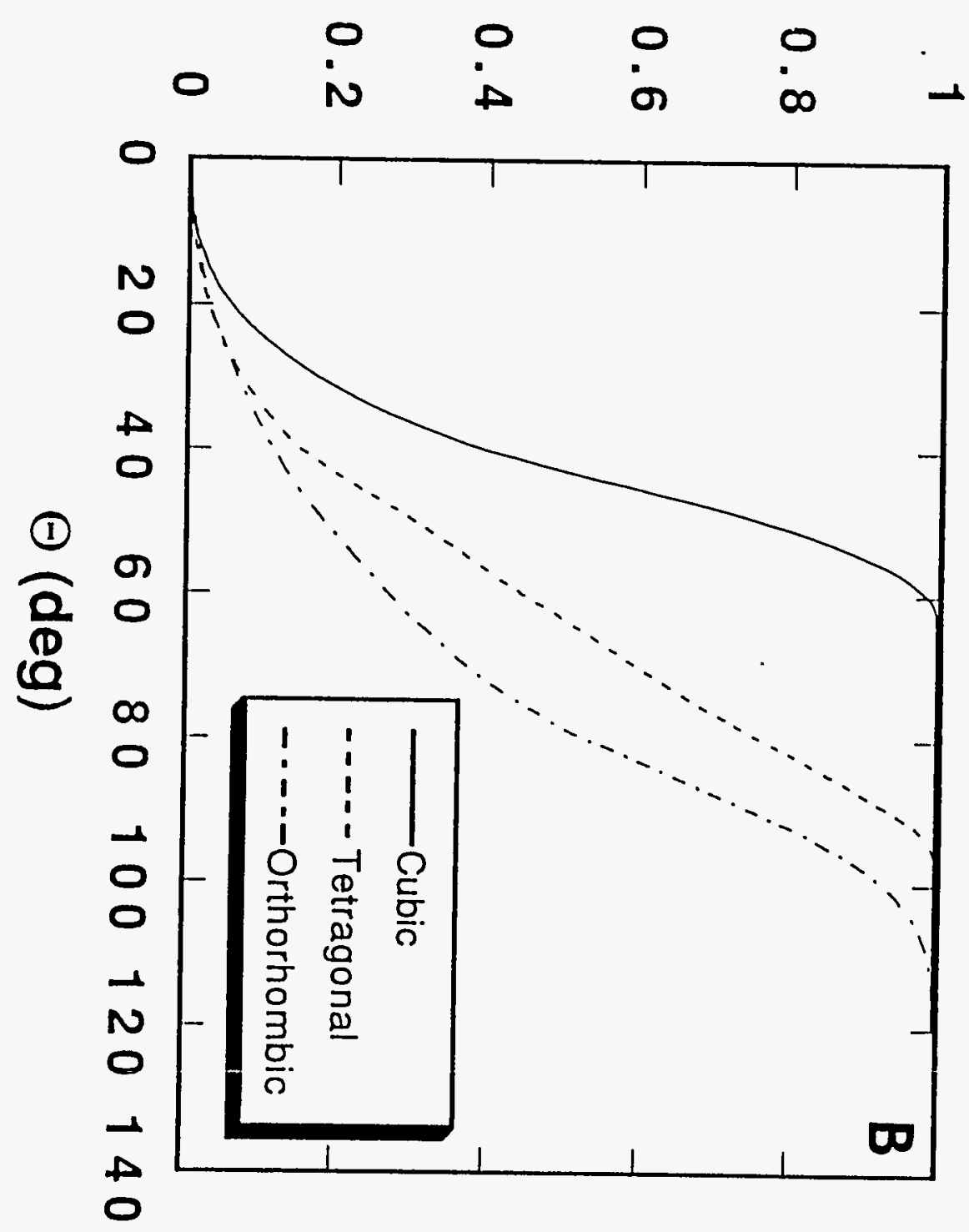

No. of Observations

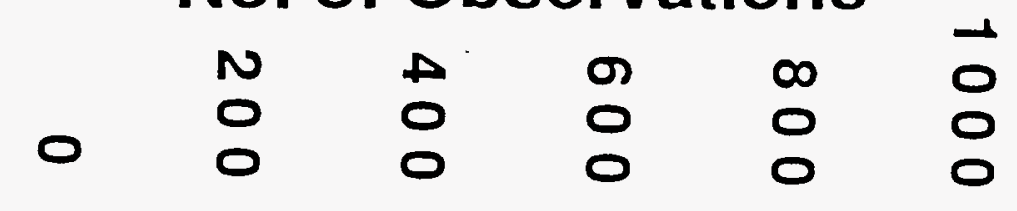

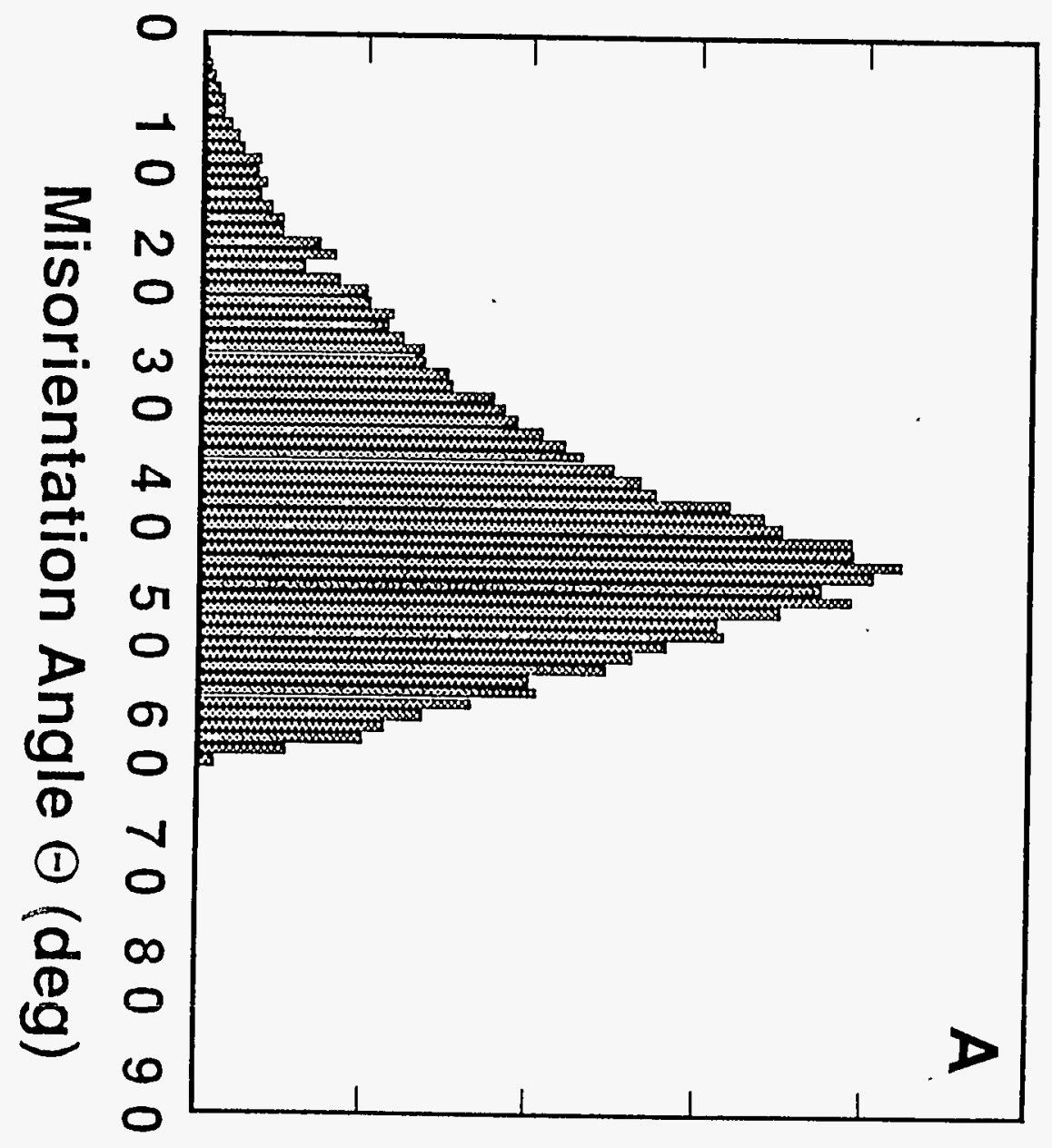




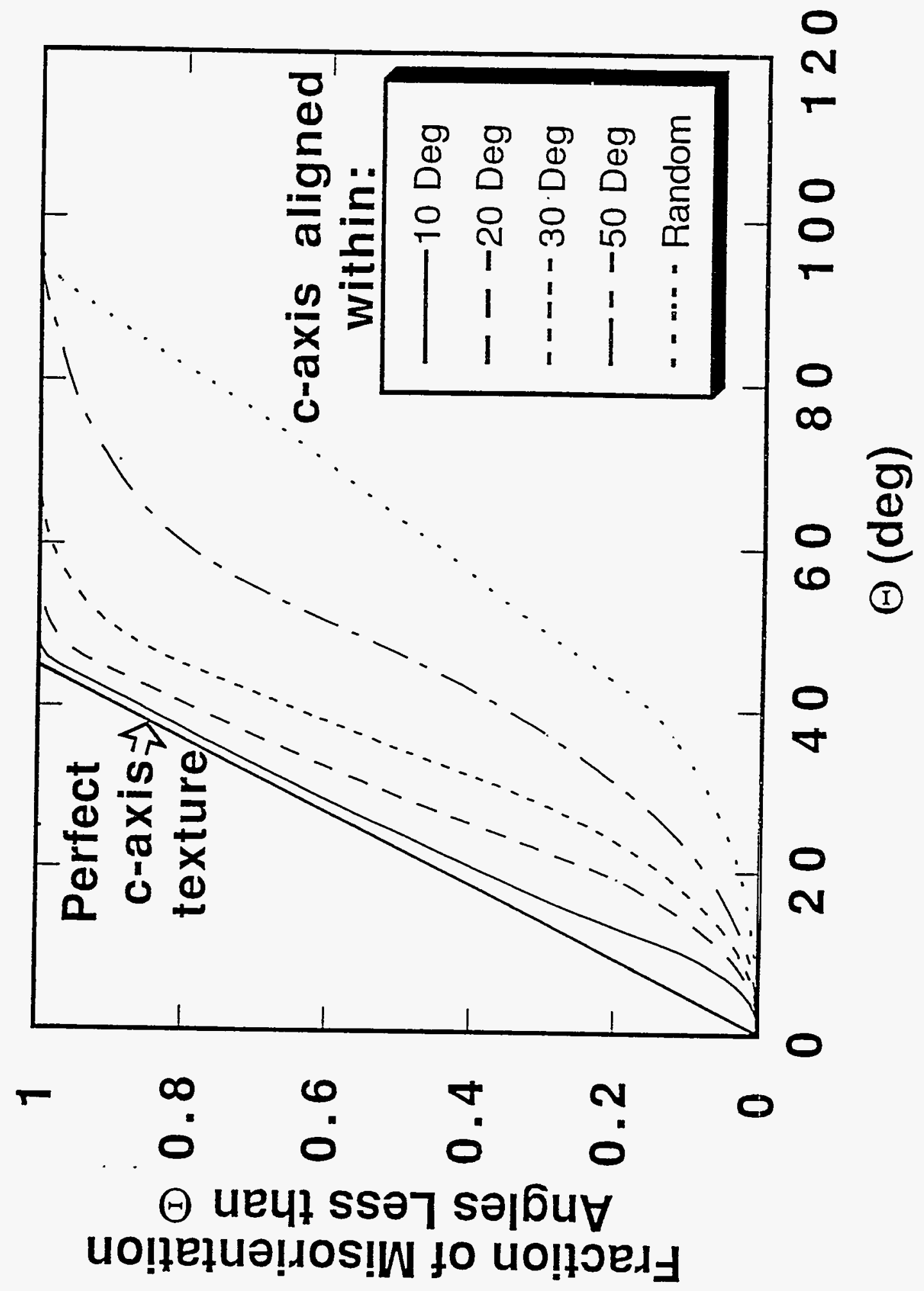




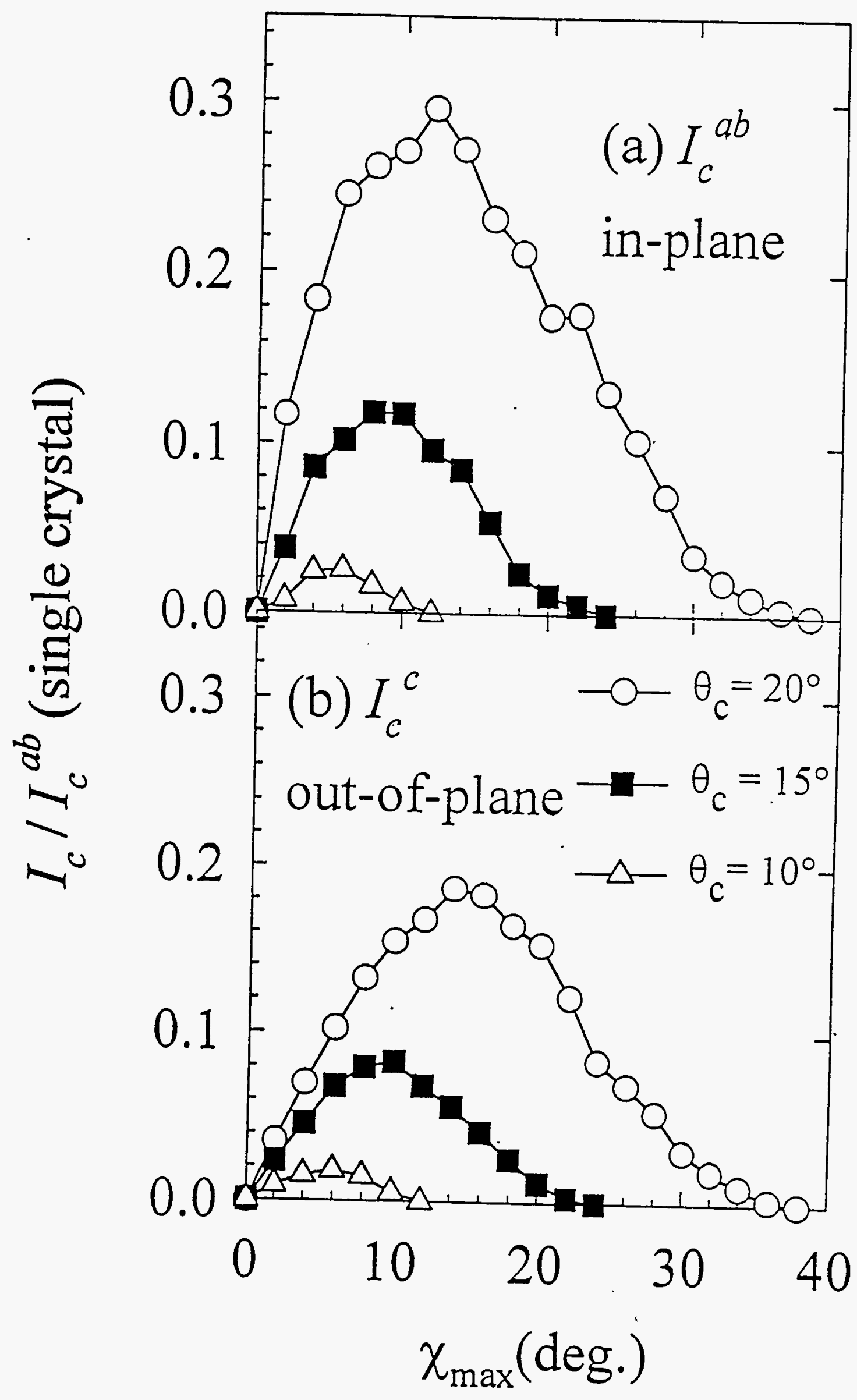




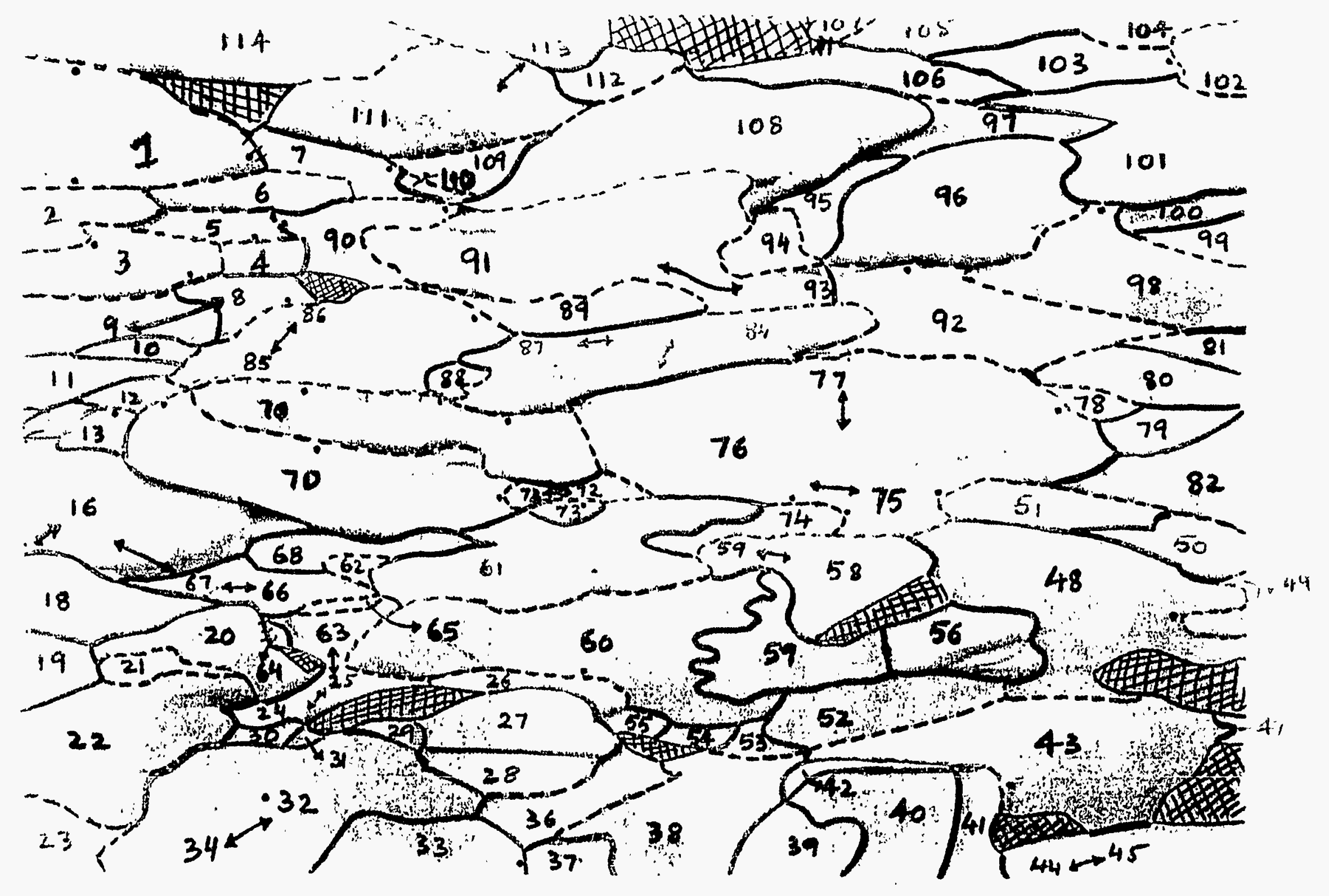




\section{Frequency}

$\%$ of Total Boundaries

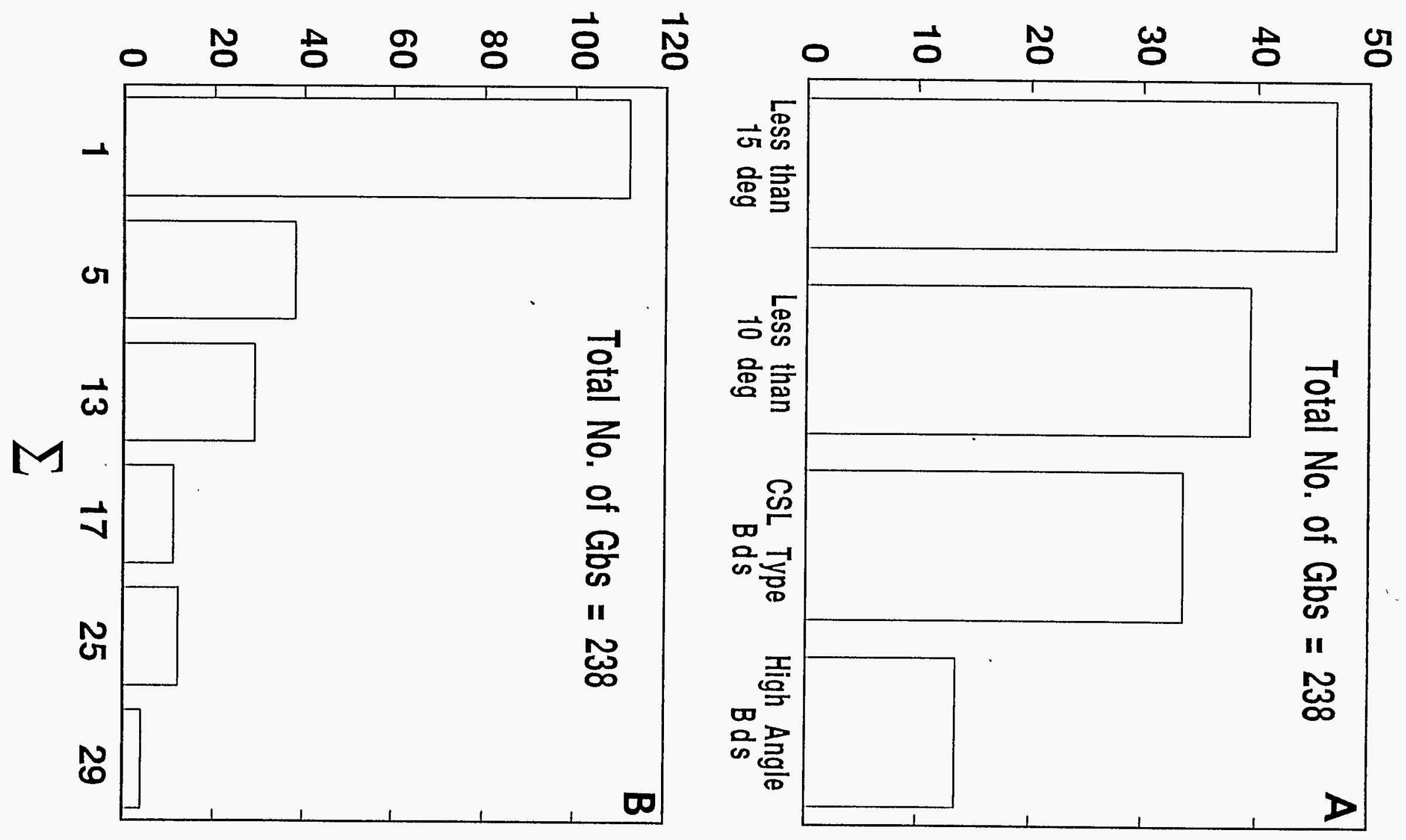




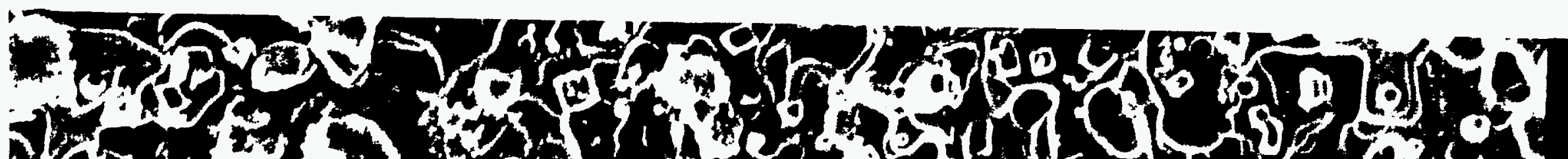

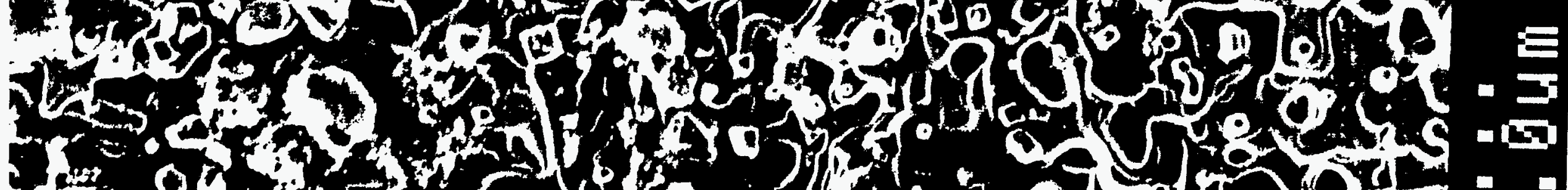
2.

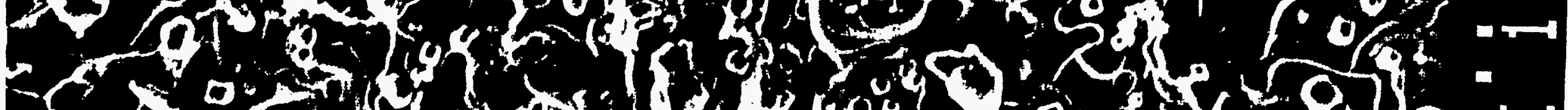

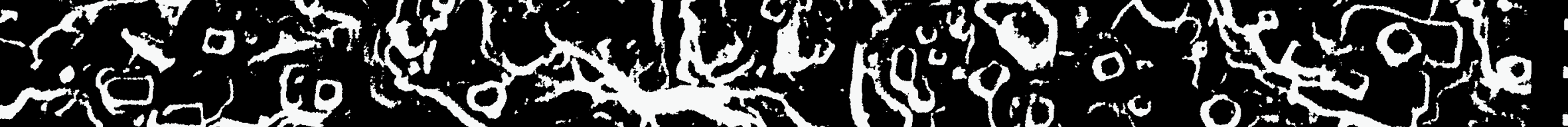

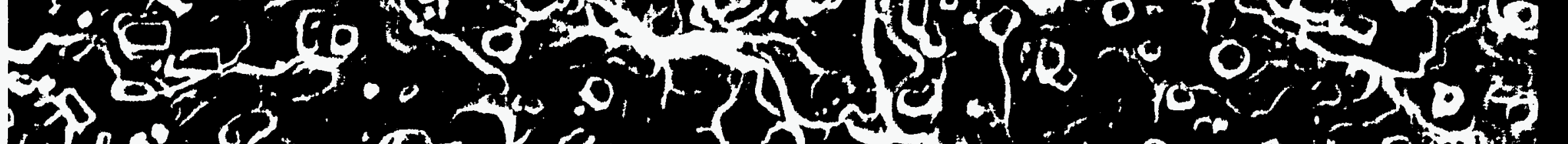

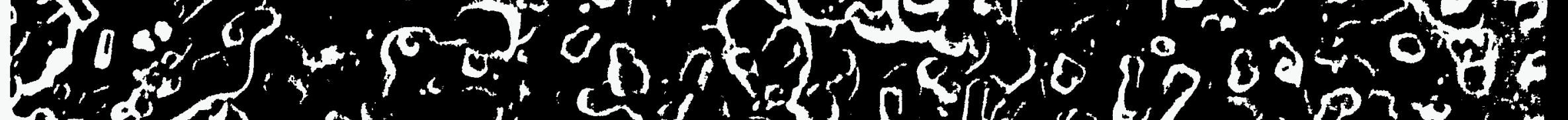

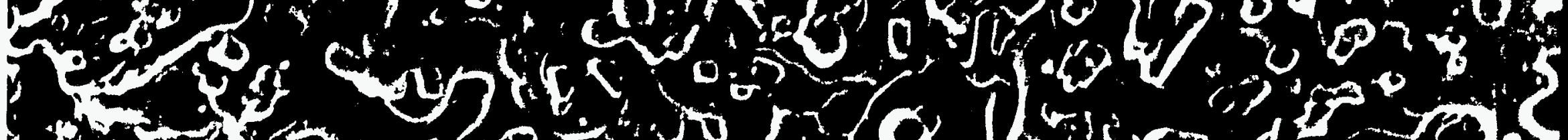

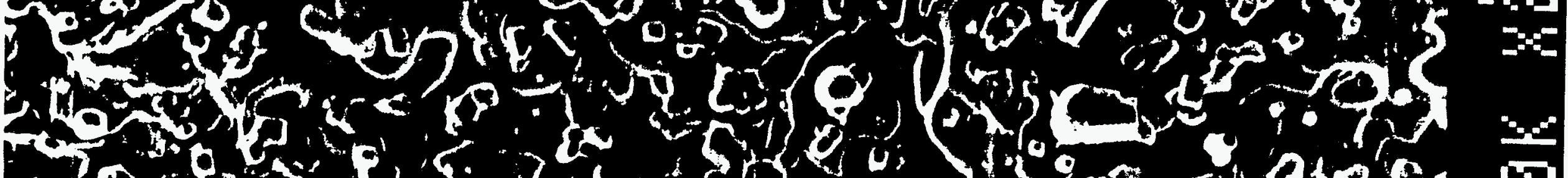

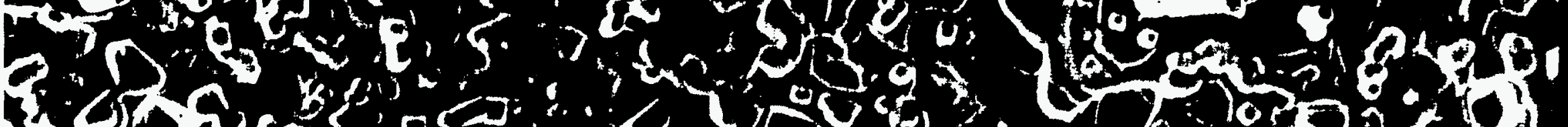

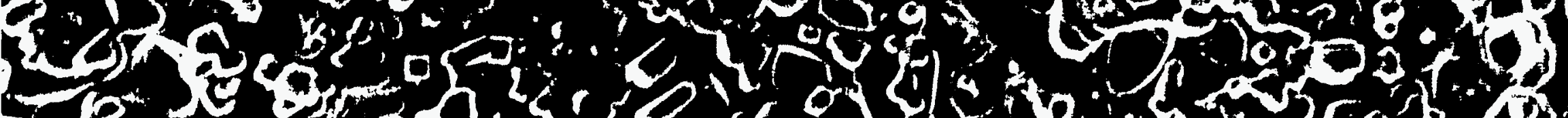

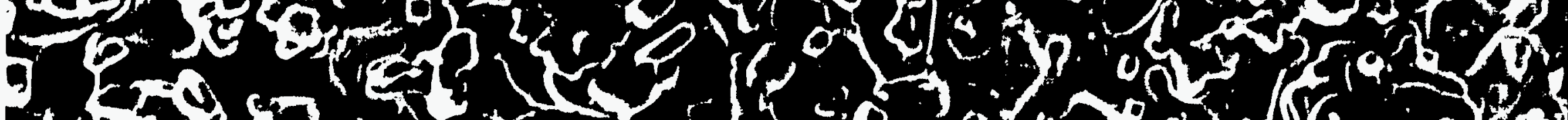
Lay a t o o P,

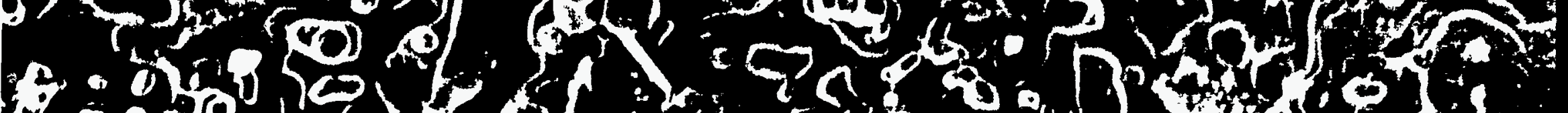

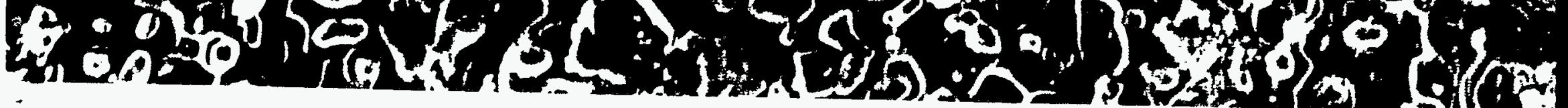
$\square$ $-17$

$-\infty$ 0 12 III "sons III 15 III in $\rightarrow$ 47 1.7. 13. 
(100) Pole Figure

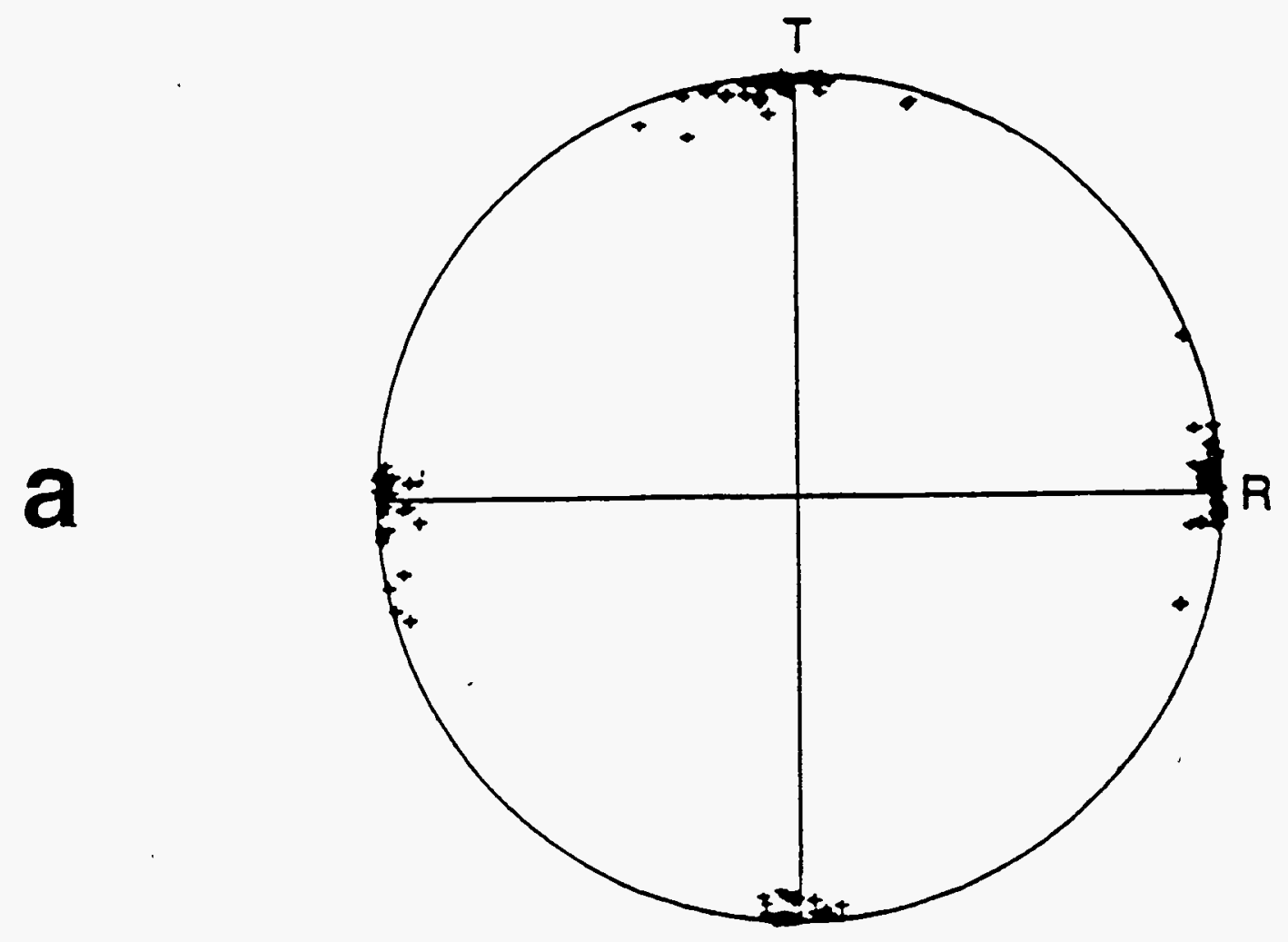

Map of a-axis Projections

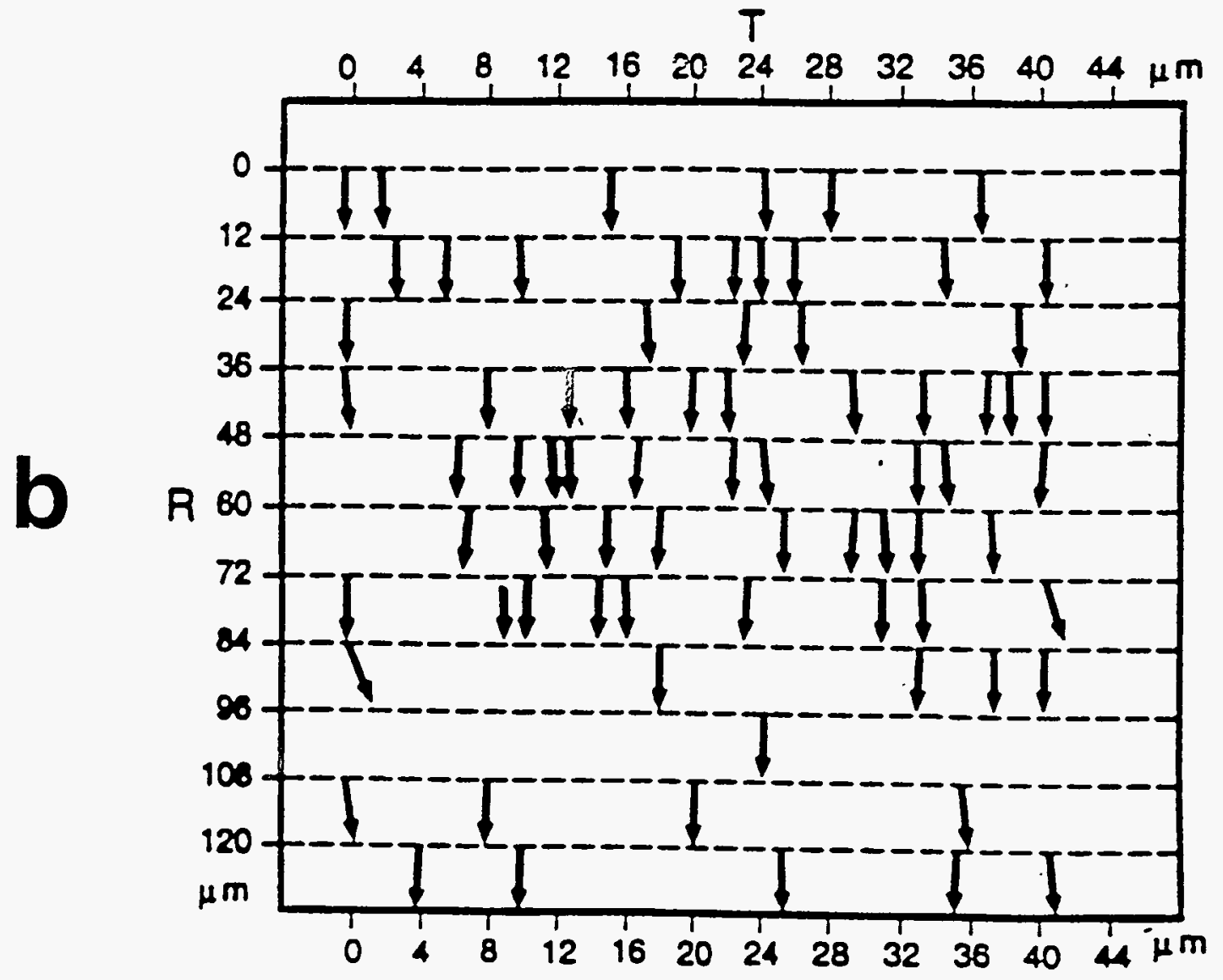


(100) Pole Figure

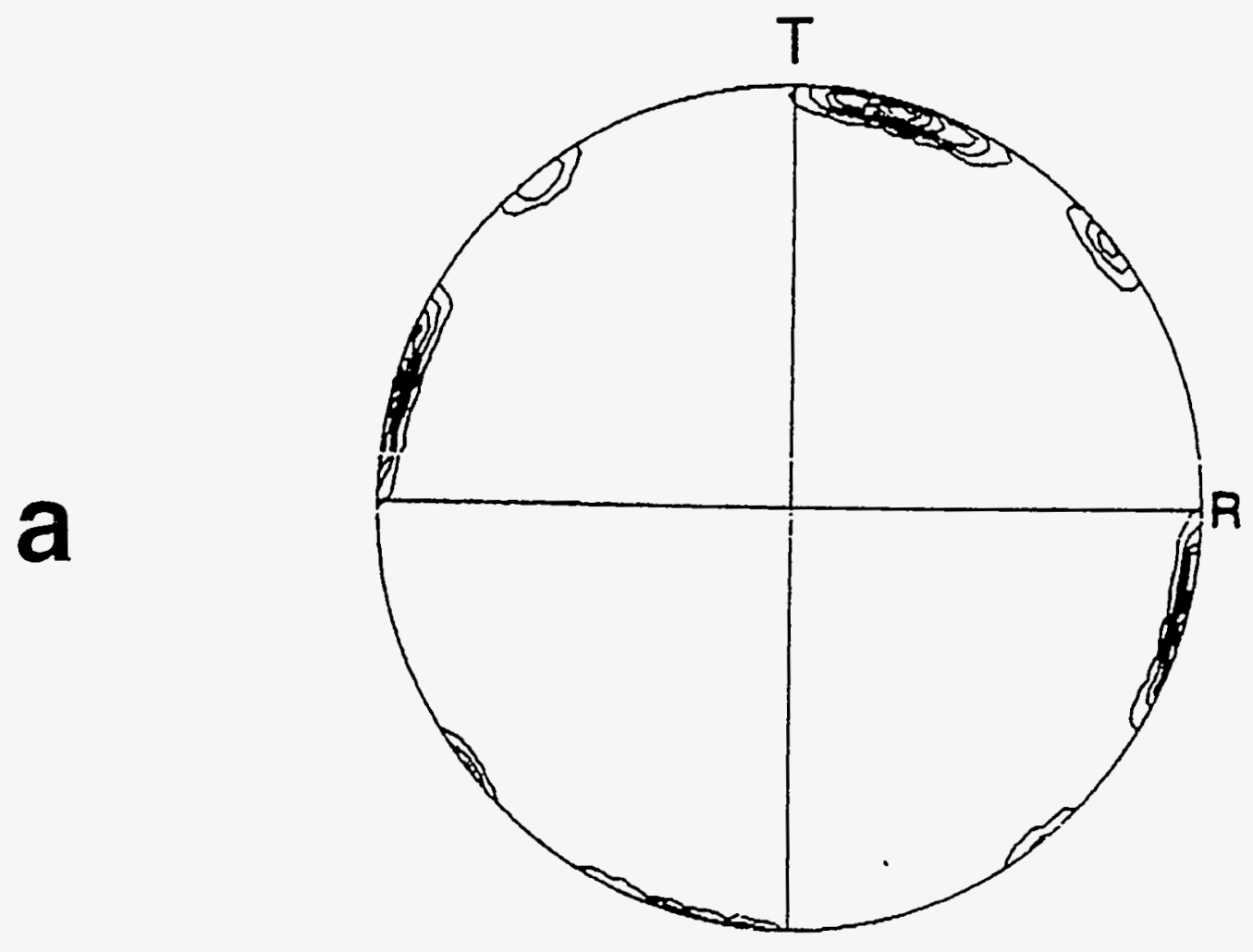

Map of a-axis Projections

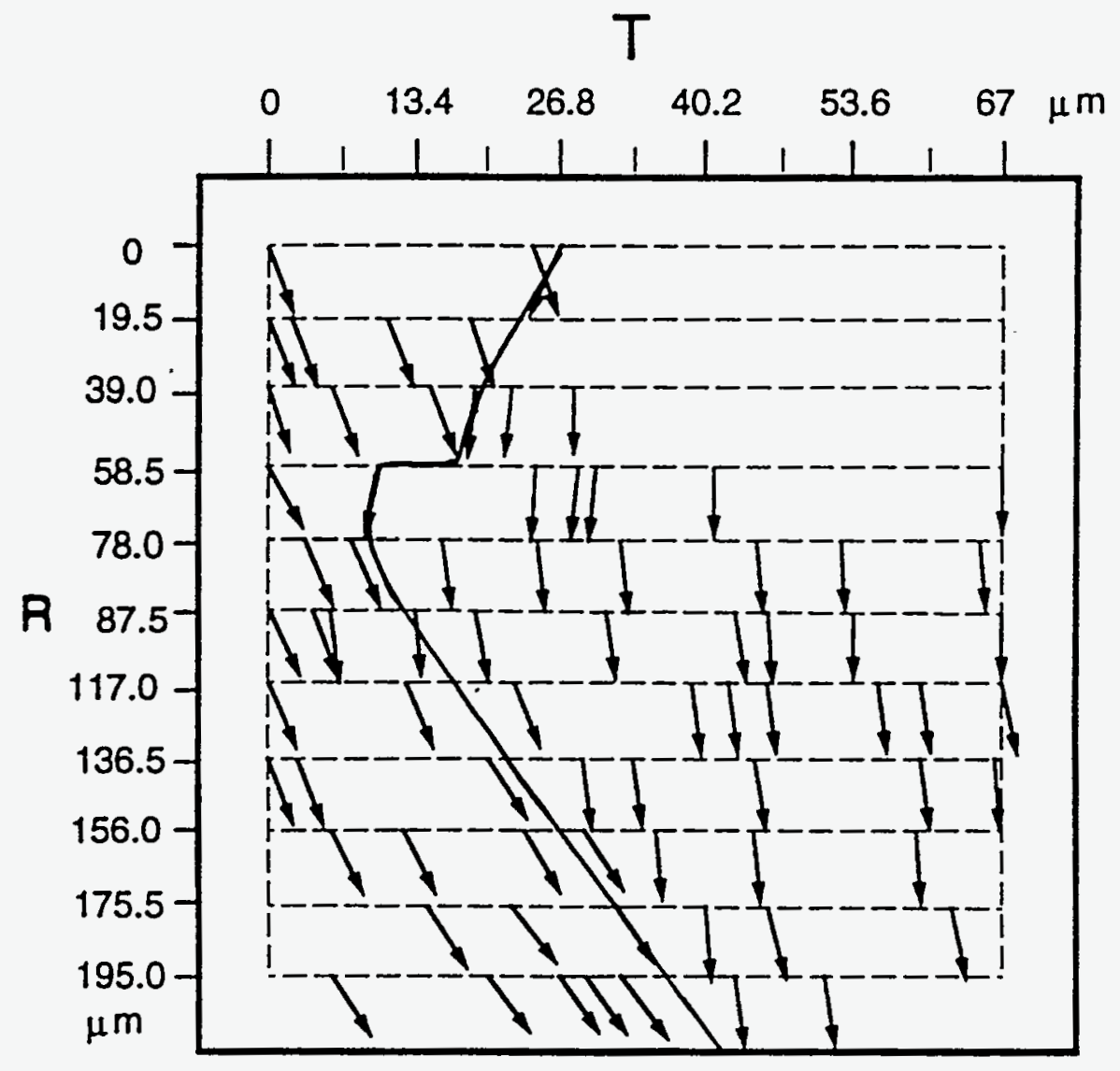

51. : 
(100) Pole Figure

a

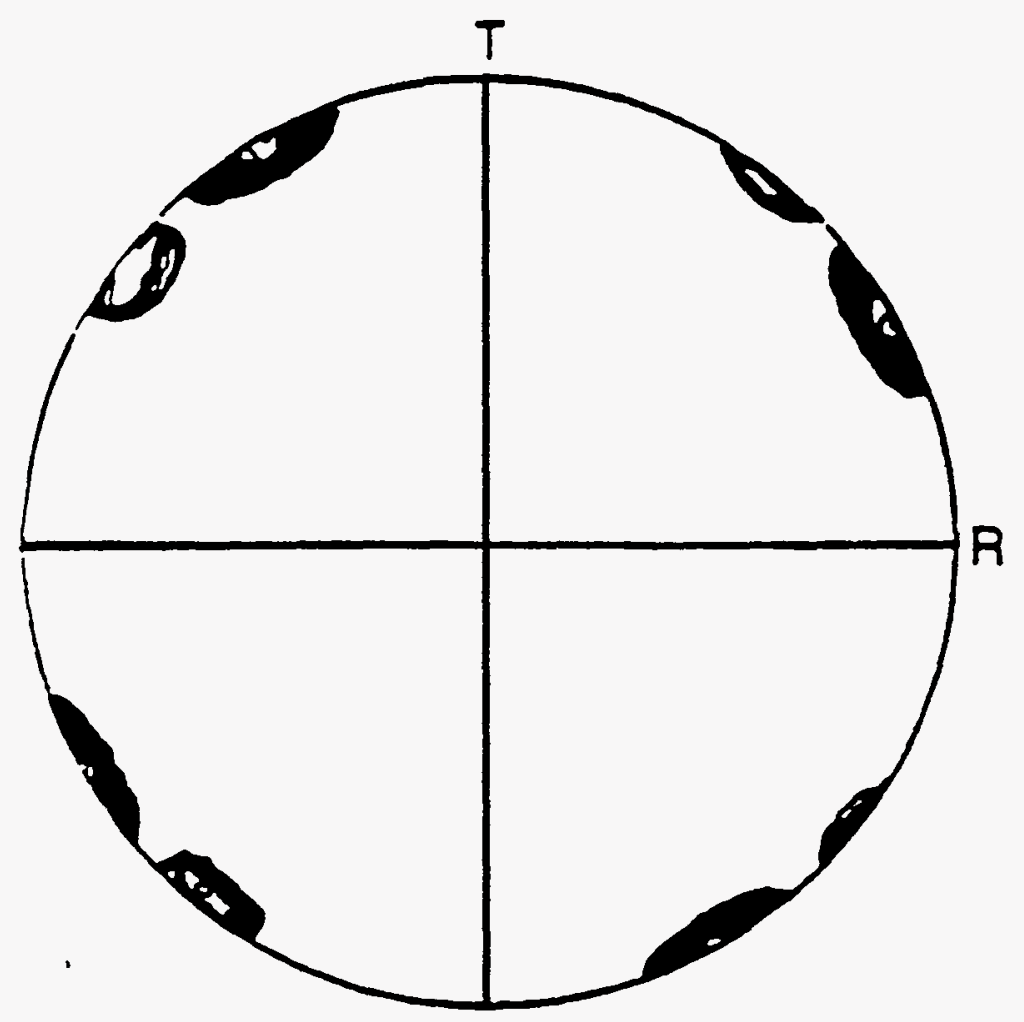

Map of a-axis Projections

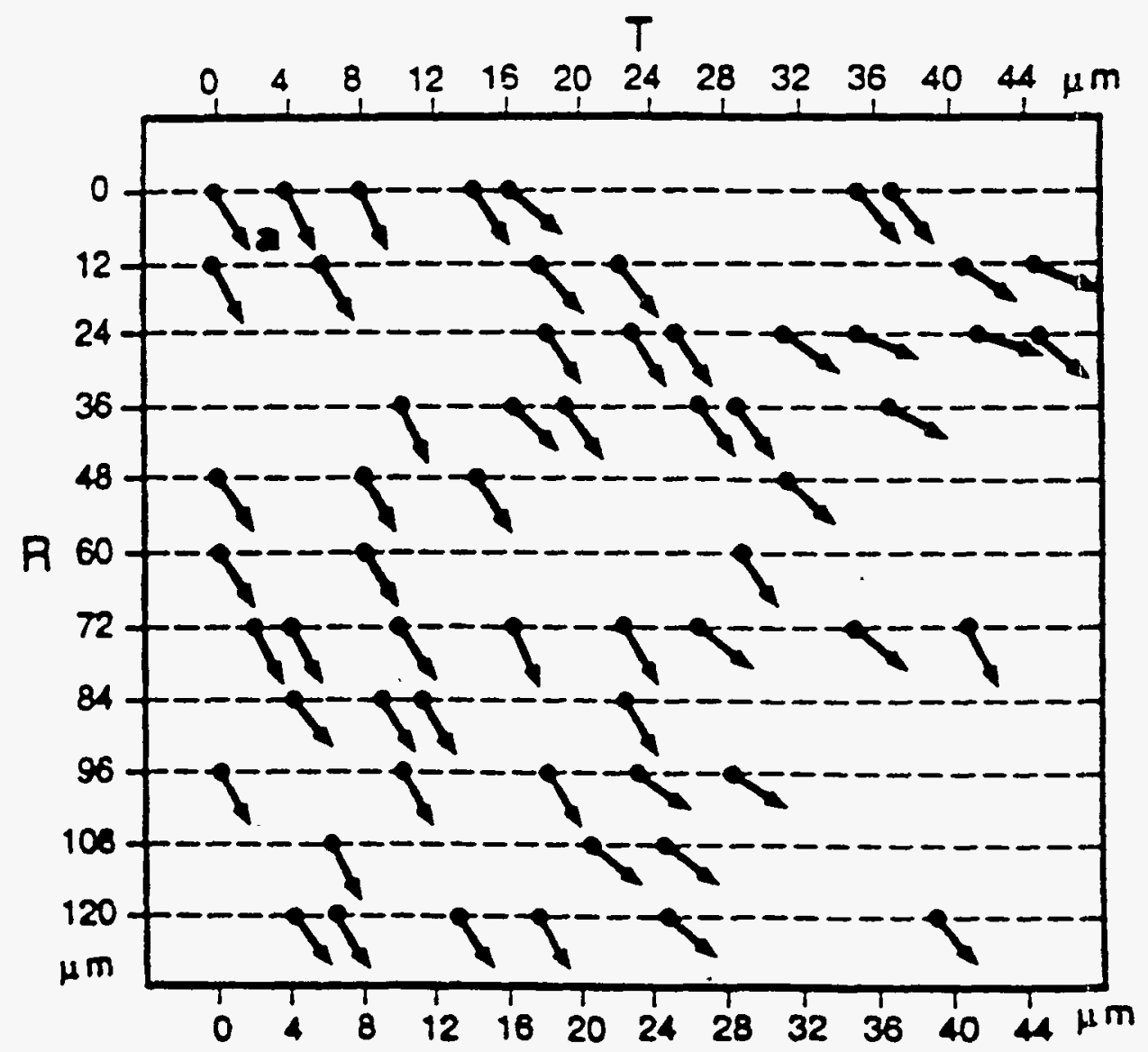


Maps of a-axis Projections in $1 \mathrm{~mm}$ regions
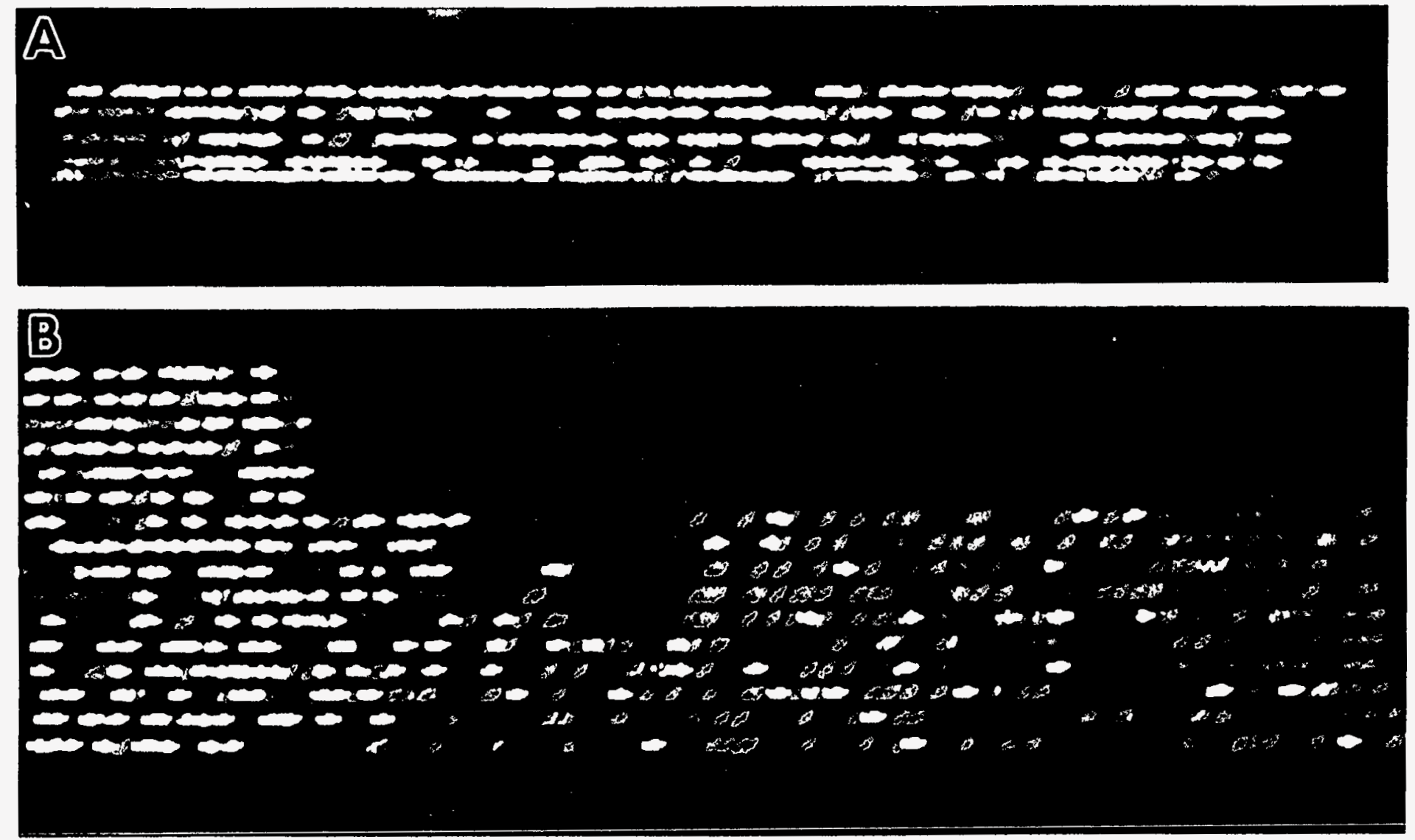

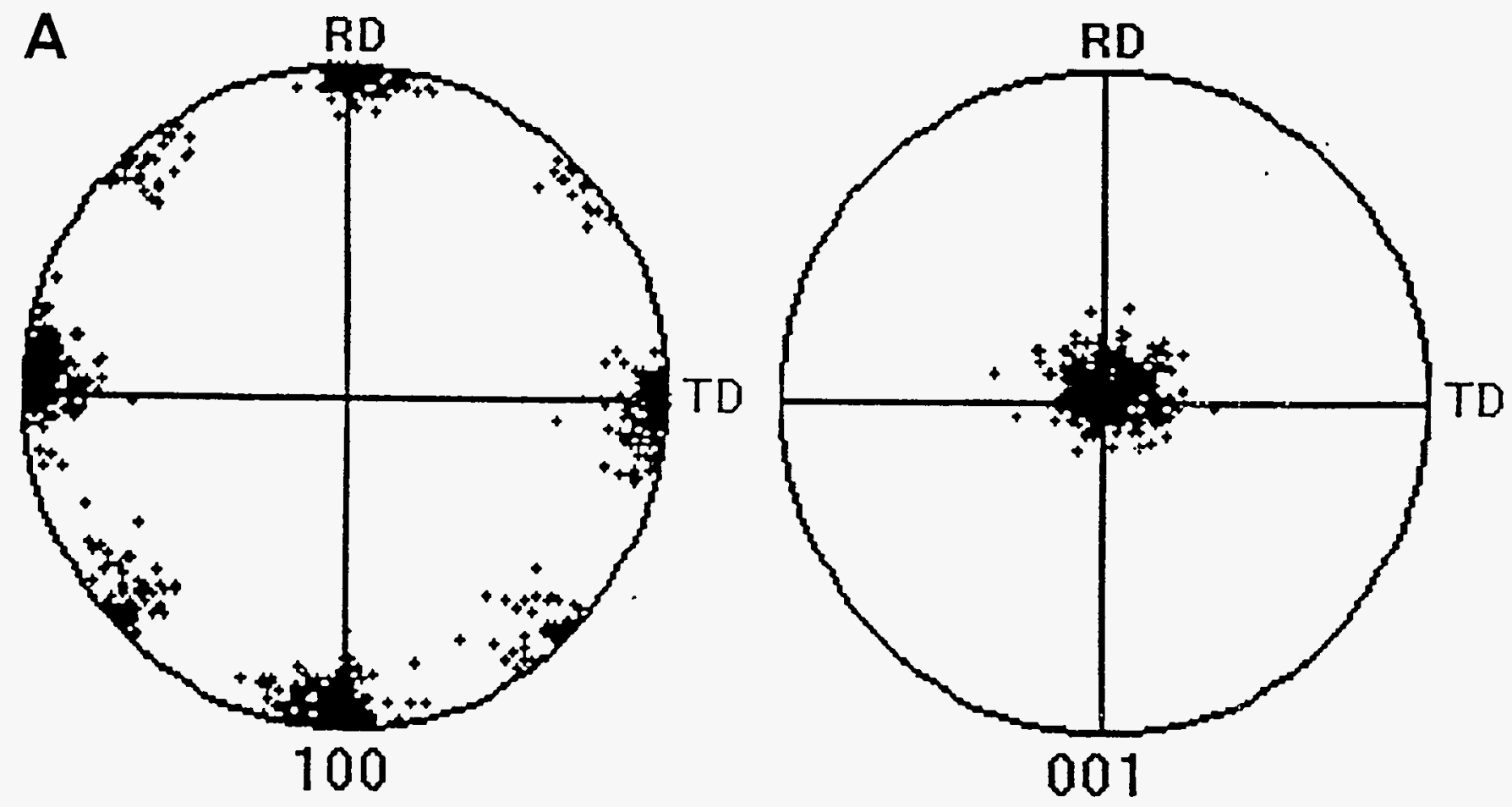

B
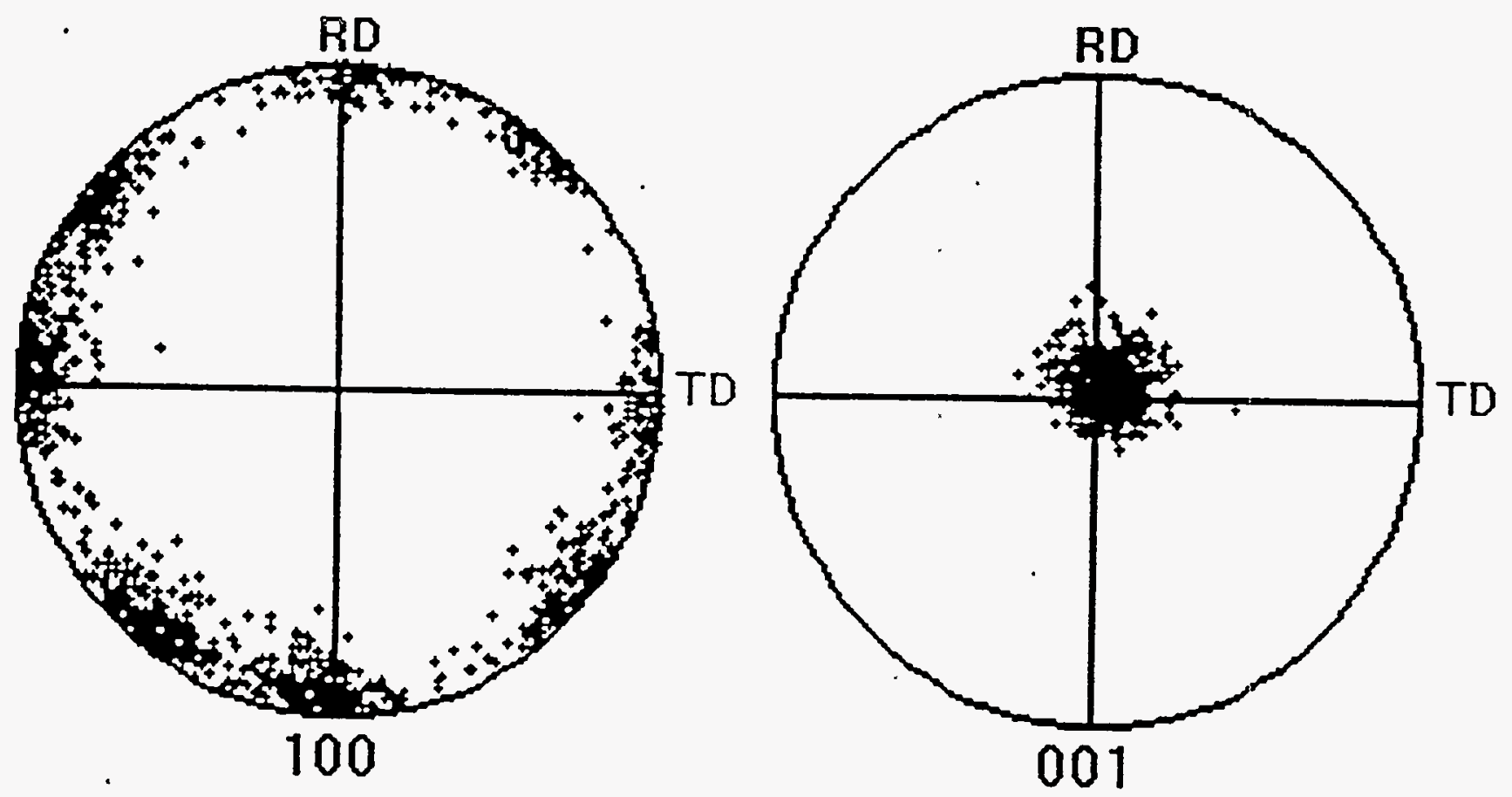

F⿻日土 8 


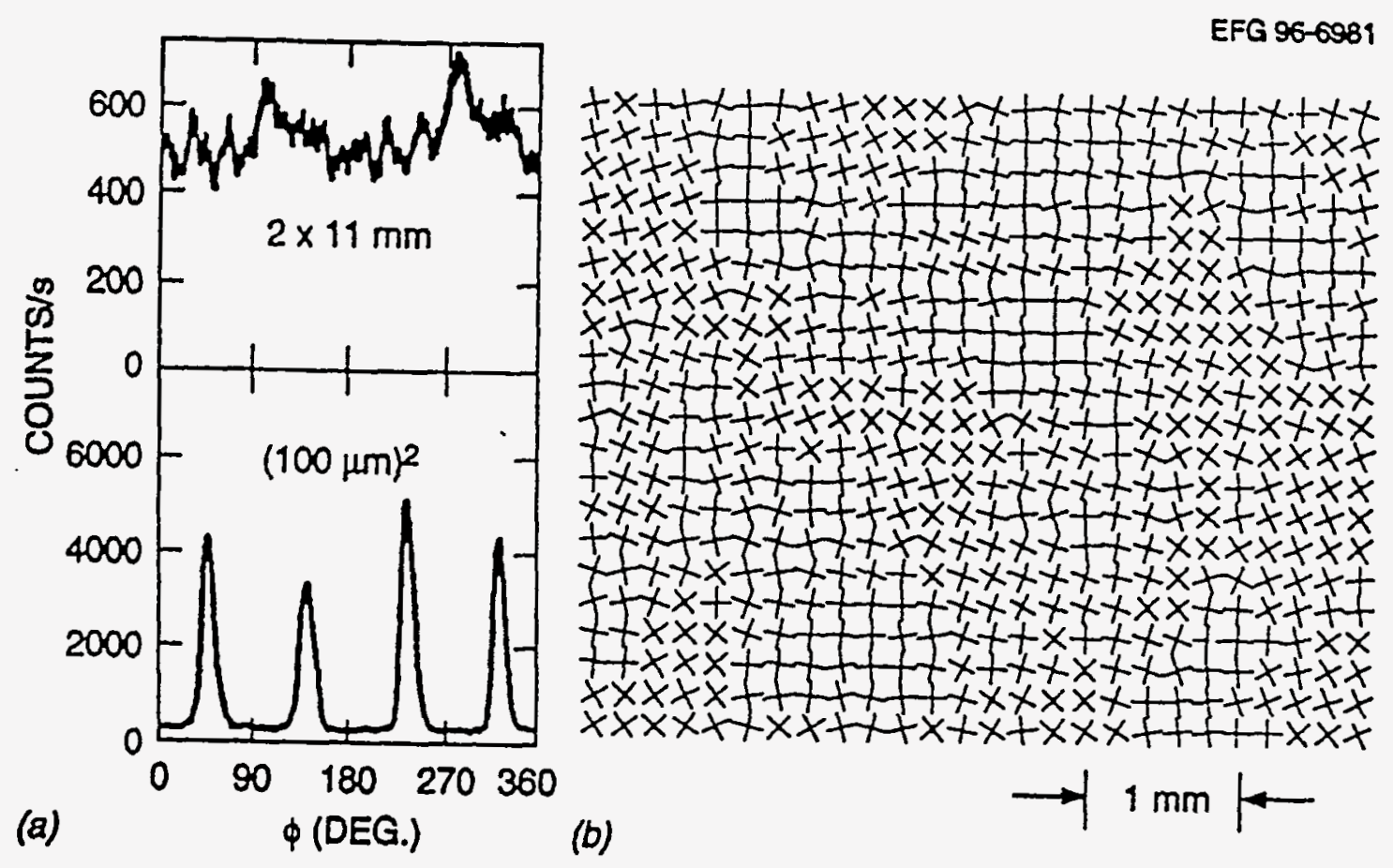

Fr. : a 


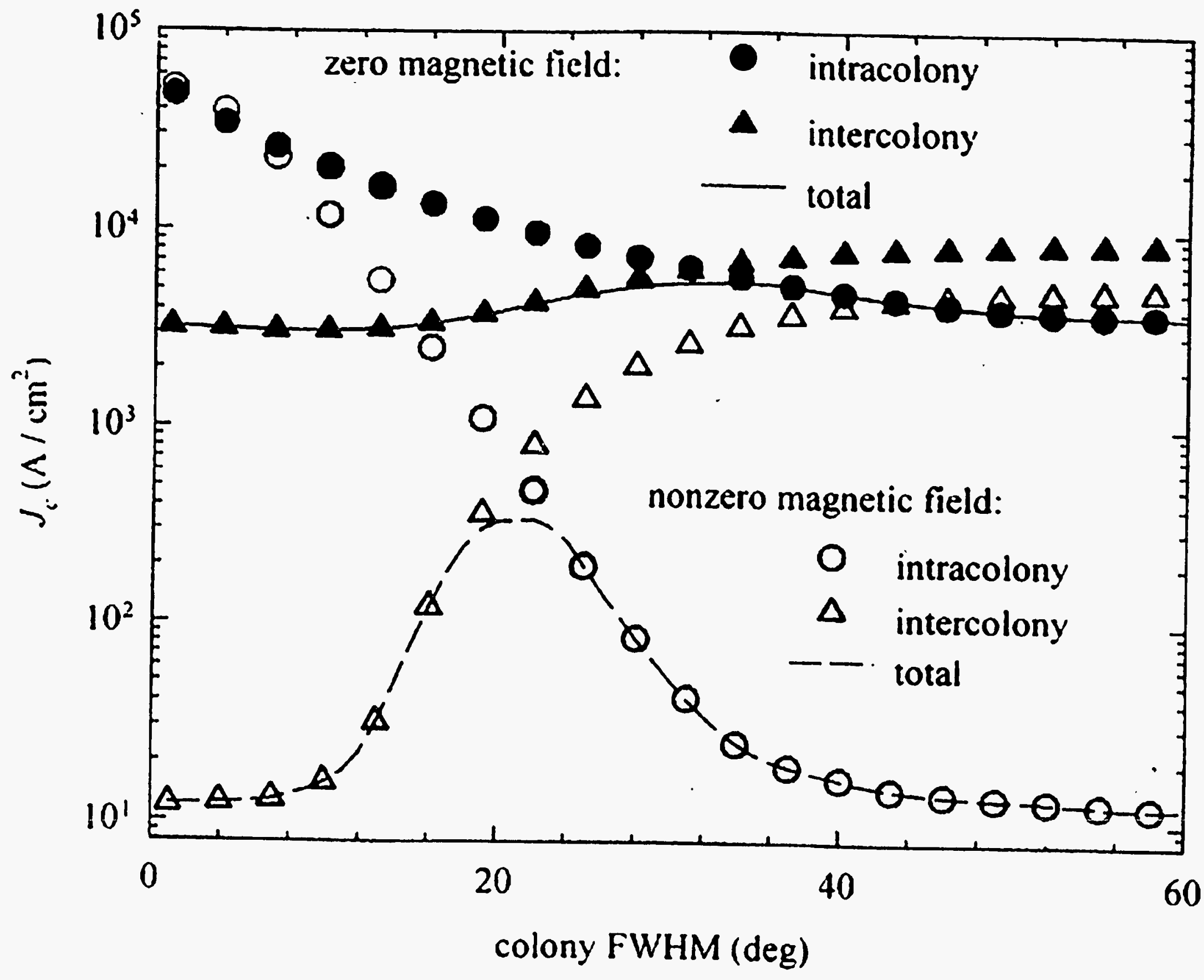




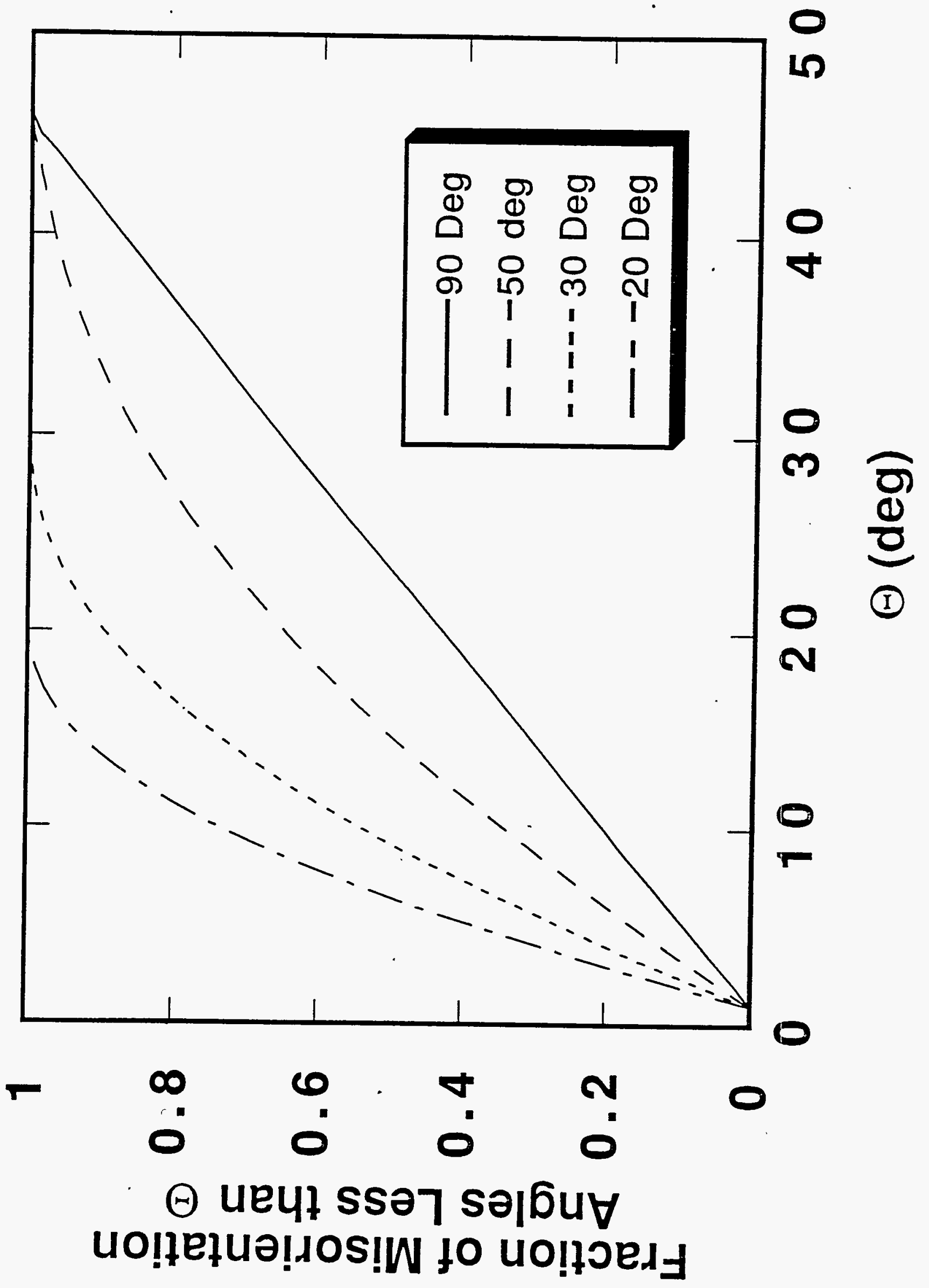




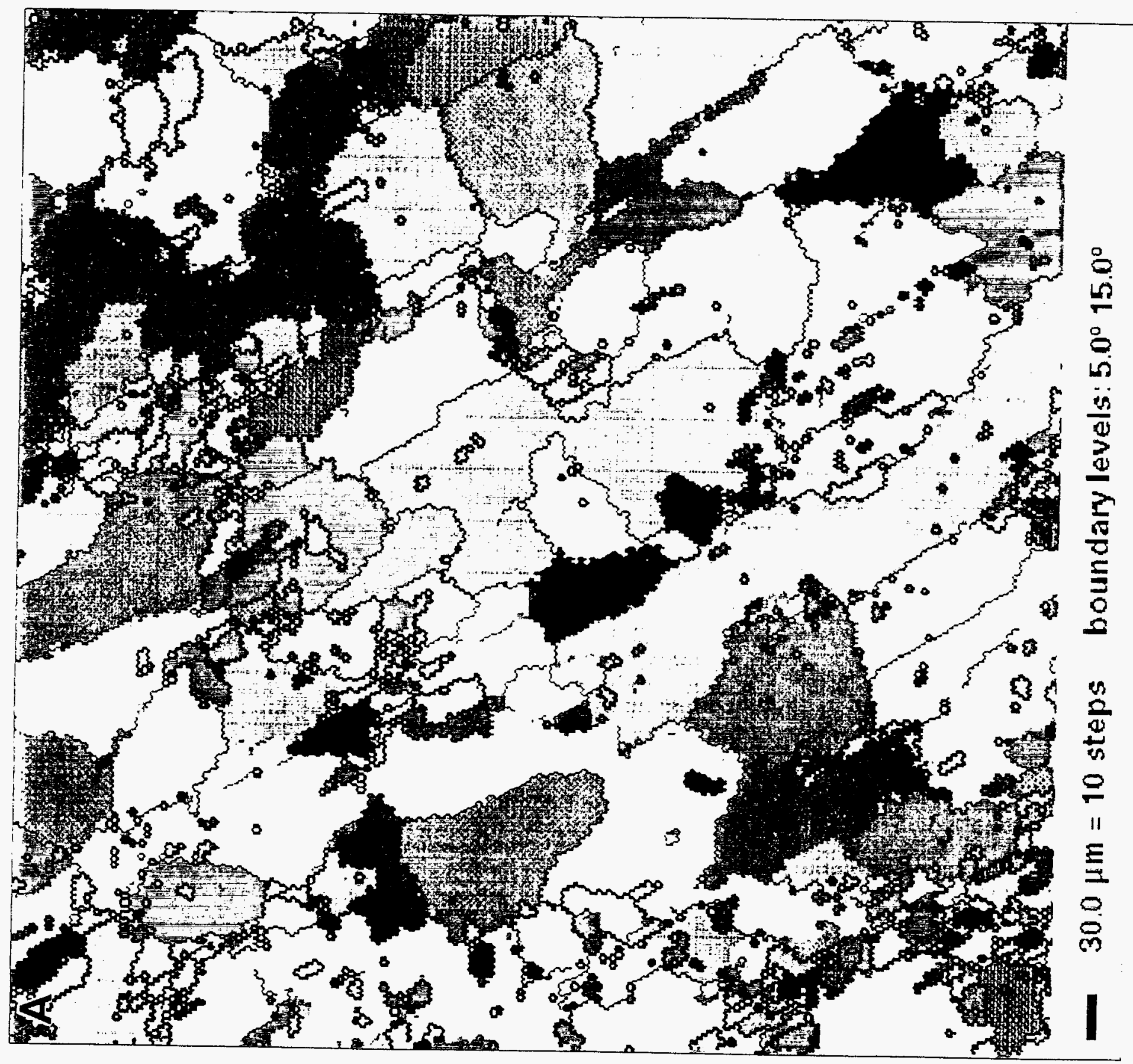




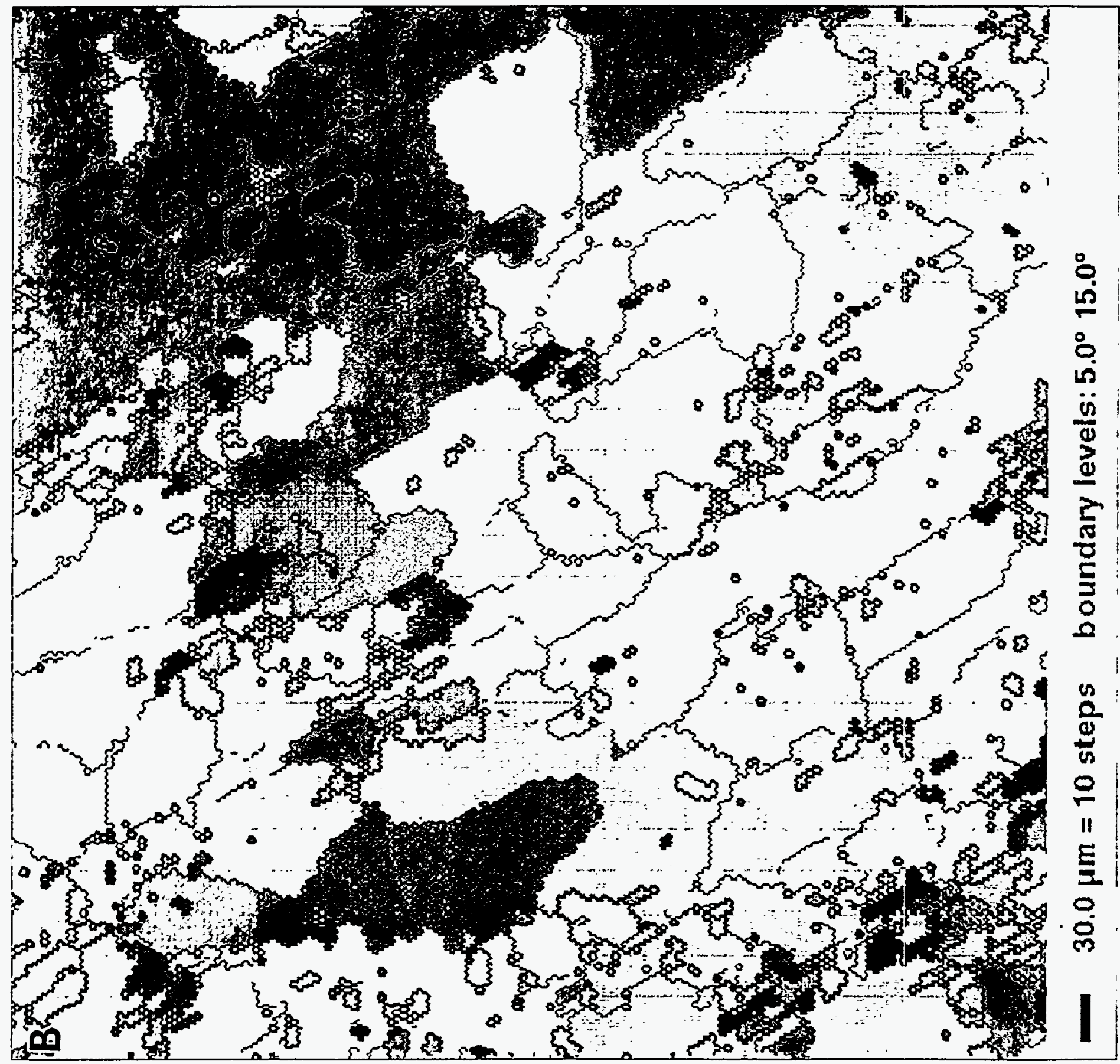




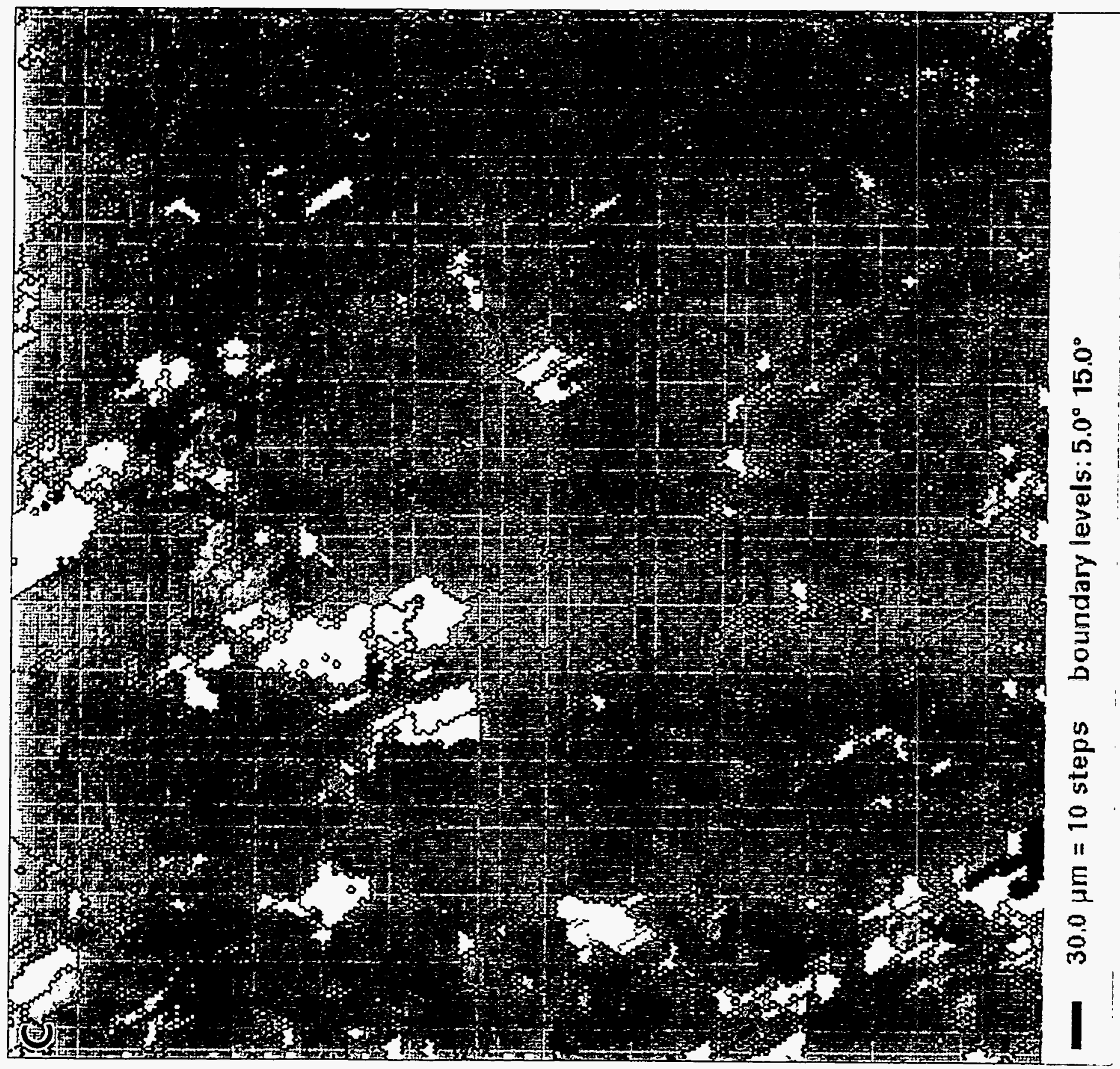

